\title{
Induced OWA Operator for Group Decision Making Dealing with Extended Comparative Linguistic Expressions with Symbolic Translation
}

\author{
Wen He ${ }^{1} \mathbb{D}$, Bapi Dutta ${ }^{2} \mathbb{D}$, Rosa M. Rodríguez ${ }^{1} \mathbb{D}$ and Ahmad A. Alzahrani ${ }^{3} \mathbb{D}$ and Luis Martínez ${ }^{1, * \mathbb{D}}$ \\ 1 Department of Computer Science, University of Jaén, 23071 Andalucía, Spain; whe@ujaen.es (W.H.); \\ rmrodrig@ujaen.es (R.M.R.) \\ 2 The Logistics Institute Asia Pacific, National University of Singapore, 21 Heng Mui Keng Terrace, \\ Singapore 119613, Singapore; tlibd@nus.edu.sg \\ 3 Faculty of Computing and Information Technology, King Abdulaziz University, Jeddah 21589, Saudi Arabia; \\ aalzahrani8@kau.edu.sa \\ * Correspondence: martin@ujaen.es
}

Citation: He, W.; Dutta, B.; Rodríguez, R.M.; Alzahrani, A.A.; Martínez, L. Induced OWA Operator for Group Decision Making Dealing with Extended Comparative Linguistic Expressions with Symbolic Translation. Mathematics 2021, 9, 20. https://dx.doi.org/10.3390/ math 9010020

Received: 28 November 2020 Accepted: 17 December 2020 Published: 23 December 2020

Publisher's Note: MDPI stays neutral with regard to jurisdictional claims in published maps and institutional affiliations.

Copyright: () 2020 by the authors. Licensee MDPI, Basel, Switzerland. This article is an open access article distributed under the terms and conditions of the Creative Commons Attribution (CC BY) license (https:/ / creativecommons.org/ licenses/by/4.0/).

\begin{abstract}
Nowadays, decision making problems have increased their complexity and a single decision maker cannot handle these problems, with a more diverse and comprehensive view of them being necessary, which results in group decision making (GDM) schemes. The complexity of GDM problems is often due to their inherent uncertainty that is not solved just by using a group. Consequently, different methodologies has been proposed to handle it, in which, the use of the fuzzy linguistic approach stands out. Among the multiple fuzzy linguistic modeling approaches, Extended Comparative Linguistic Expressions with Symbolic Translation (ELICIT) information has been recently introduced, which enhances classical linguistic modeling that is based on single terms by providing linguistic expressions in a continuous linguistic domain. Its application to decision making is quite promising, but it is necessary to develop enough operators to accomplish aggregation processes in the decision solving scheme. So far, just a small number of aggregation operators have been defined for ELICIT information. Hence, this paper aims at providing new aggregation operators for ELICIT information by developing novel OWA based operators, such as the Induced OWA (IOWA) operator in order to avoid the OWA operator needs of reordering its arguments, because ELICIT information does not have an inherent order due to its fuzzy representation. Our proposal not only consists of extending the definition of an IOWA operator for ELICIT information with crisp weights, but it is also proposed a type-1 IOWA operator for ELICIT information in which both weights and arguments are fuzzy as well as the use of ELICIT information constructing the order inducing variable to reorder the arguments. Additionally, the use of ELICIT information in GDM demands the ability to manage majority based decisions that are better represented in the IOWA operator by linguistic quantifiers. Hence, a majority-driven GDM process for ELICIT information is proposed, which it is the first proposal for fulfilling the majority solving process for GDM while using ELICIT information. Eventually, an illustrative example and a brief comparative analysis are presented in order to show the performance of the proposal and its feasibility.
\end{abstract}

Keywords: aggregation operators; computing with words; ELICIT information; group decision making

\section{Introduction}

Decision making is an everyday life activity for human beings that range from simple to very complex problems. The increasing complexity of decision making problems that face companies, organizations, and decision makers has made necessary the development of comprehensive frameworks in which multiple views and knowledge about the problem are included [1]. Such a type of problems conform the group decision making (GDM) scheme [2], in which collective solutions are chased to make the decision and they are 
usually defined under high uncertain contexts [3]. To handle such an uncertainty, multiple proposals have been developed in the specialized literature for modeling decision makers' preferences uncertainties [3-7], in which fuzzy sets theory and the fuzzy linguistic approach have provided successful results in multiple applications to decision making under uncertainty [8-10].

The application of fuzzy linguistic variables to decision making has evolved from single linguistic terms [11-14] to Extended Comparative Linguistic Expressions with Symbolic Translation (ELICIT) [15], in which linguistic expressions that are conformed by multiple linguistic terms representing a continuous domain are used for assessing preferences. In the middle, the use of linguistic 2-tuple [9,16], Hesitant Fuzzy Linguistic Term Sets (HFLTSs) [17], Comparative Linguistic Expressions (CLEs), etc., have given support to developing a big tool box for managing uncertainty in decision making. In all previous cases, the linguistic preference modeling implied the need of operating with linguistic values that have been accomplished by the Computing with Words (CW) methodology $[18,19]$.

Among the different necessary linguistic computations for decision making, the aggregation operators play a key role in fusing information that comes from different sources into a single information, which represents an overall overview [20]. Most of the previous linguistic preference modeling approaches have developed their correspondent fuzzy computational model with multiple extended aggregation operators for aggregating information during the decision processes. However, the ELICIT approach [15] due to its youth there still needs to develop more aggregation operators, because, so far, just a few number of them have been specifically defined to aggregate ELICIT expressions, namely, the fuzzy arithmetic mean [15] and the Bonferroni mean [21]. Hence, in this paper, we aim at developing several aggregation operators to aggregate ELICIT information by capturing majority opinion that has been expressed in this context by means of the fuzzy linguistic quantifier "most". The notion of quantifier guided aggregation has been formally defined by means of ordered weighted averaging (OWA) operators [22-24] and by means of the concept of fuzzy integrals $[3,20,25]$. Our focus on this study is the use of OWA based operators for ELICIT information. In particular, we will focus on the induced OWA (IOWA) operator [26,27], because, among the other advantages, the reordering of arguments, which is required by the family of OWA operators, is based on the order inducing variable, and, in this way, we do not need in a compulsory way to rank fuzzy numbers as the ones that represent the ELICIT information. Consequently, different IOWA operators for ELICIT information will be introduced in this research:

(i) According to Zadeh's extension principle, an IOWA operator is extended to ELICIT information with the crisp weight, the so-called ELICIT-IOWA operator. Generally, the weight is computed by the linguistic quantifier that is expressed by the basic unit-interval monotonic (BUM) function [28]. However, if the argument variables have associated importance, the BUM function is used to obtain the important ELICIT-IOWA (ELICIT-I-IOWA) operator. Simultaneously, when considering that the ELICIT expression can be equivalently converted into a trapezoidal fuzzy number [15], by adding the continuous monotonic function $g$ to the ELICIT-IOWA operator, we will obtain a wide range of ELICIT-IOWA operators.

(ii) When considering the type-1 OWA operator, the induced type-1 OWA operator with fuzzy weights, so-called the t1-IOWA operator, will be introduced. Using Zadeh's expansion principle, the t1-IOWA operator is extended to ELICIT information in order to obtain the ELICIT-t1-IOWA operator.

(iii) Eventually, it will be proved that both the ELICIT-IOWA and ELICIT-t1-IOWA operators have the general properties of the IOWA operator, because they are both IOWA-based operators.

Additionally, as shown in [29], the IOWA operator is adequate for modeling the majority driven opinions by using fuzzy linguistic quantifiers, such as "most", "as many as possible", and "at least half", to manage the degree of majority. Based on these linguistic 
quantifiers and the ELICIT-IOWA based aggregation operators, we will propose a majoritydriven method for solving the GDM problem dealing with ELICIT information.

The paper is set out as follows: Section 2 revises related concepts of IOWA operators, fuzzy linguistic quantifiers, and ELICIT information. Section 3 proposes different ELICITIOWA operators. Section 4 proves the general properties of the previous ELICIT-IOWA operators. Section 5 provides a majority driven GDM scheme for ELICIT information. Section 6 provides an illustrative example to show the performance and feasibility of the method that is based on the ELICIT-IOWA operator and briefly introduces the comparison among other linguistic quantifiers. Finally, Section 7 concludes the paper.

\section{Preliminaries}

This section revises key concepts related to the induced OWA (IOWA) operator, fuzzy linguistic quantifiers, and ELICIT information.

\subsection{The Induced Owa Operator}

The OWA operator was defined by Yager [30], which is a mapping OWA $: \mathbb{R}^{n} \longrightarrow$ $\mathbb{R}$ that has an associated weighting vector $W$ of dimension $n$ with the properties $\omega_{i} \in$ $[0,1], i=1, \cdots, n$ and $\sum_{j=1}^{n} \omega_{i}=1$, such that

$$
\operatorname{OWA}\left(a_{1}, a_{2}, \cdots, a_{n}\right)=\sum_{i=1}^{n} \omega_{i} a_{\sigma(i)}
$$

where $\mathbb{R}$ be the set of all real numbers and $\sigma:\{1, \cdots, n\} \longrightarrow\{1, \cdots, n\}$ is a permutation function, such that $a_{\sigma(i)}$ is the $i^{\text {th }}$ largest element in the set $\left\{a_{1}, \cdots, a_{n}\right\}$.

Because the real set $\mathbb{R}$ has an inherent order, so that, when considering the weight that is associated to the ordered position to produce the family of the OWA operator [24,28], for instance, the max OWA operator is guided by $W^{*}=(1,0, \cdots, 0)$, the min OWA operator with $W_{*}=(0, \cdots, 0,1)$, and the continuous OWA (C-OWA) operator [31] applies the OWA operator to a continuous interval $[a, b]$ with crisp weights that are computed by a BUM function $Q$ [28] as $F_{Q}([a, b])=\int_{a}^{b} \frac{d Q(y)}{d y}(b-y(b-a)) d y$, and type-1 OWA operator [32] with both arguments and weights in the form of the type- 1 fuzzy set, and so on. However, if we want to apply the OWA operator to the fuzzy representation model, it is not straightforward, because fuzzy numbers do not have an inherent order. For instance, let us assume that the type-1 OWA operator [32] is applied to fuzzy numbers $\left\{A_{i}\right\}_{i=1}^{n}$ with fuzzy weights $\left\{\hat{w}_{i}\right\}_{i=1}^{n}$, and both $A_{i}$ and $\hat{w}_{i}$ are defined on the universe of discourse $[0,1]$. It aggregates $\left\{A_{i}\right\}_{i=1}^{n}$ by the following formula:

$$
\begin{gathered}
\mu_{G}(y)=\sup _{\substack{\sum_{i=1}^{n} \omega_{i} a_{\sigma(i)} \\
\sum_{i=1}^{n} \omega_{i}}}\left\{\mu_{\hat{w}_{1}}\left(\omega_{1}\right) * \mu_{A_{1}}\left(a_{1}\right) * \cdots * \mu_{\hat{w}_{n}}\left(\omega_{n}\right) * \mu_{A_{n}}\left(a_{n}\right)\right\} \\
\forall \omega_{i}, a_{i} \in[0,1]
\end{gathered}
$$

where $*$ is the t-norm operator and $y$ is obtained by the classical OWA operator. Because of the fact that fuzzy numbers have no inherent order, the type-1 OWA operator only uses the OWA operator in the calculation of $y=\frac{\sum_{i=1}^{n} \omega_{i} a_{\sigma(i)}}{\sum_{i=1}^{n} \omega_{i}}, \forall \omega_{i}, a_{i} \in[0,1]$ in order to avoid reordering of fuzzy numbers $\left\{A_{i}\right\}_{i=1}^{n}$. In a sense, this is not a straightforward application of the OWA operator to fuzzy numbers. Therefore, the application of classical OWA operators is constrained, especially when the argument variable has a fuzzy representation.

Therefore, it is necessary to provide a method in order to solve the reordering step of OWA operators. One method is to use the fuzzy number ranking method, and another method is to add an additional variable, the so-called the order inducing variable $u_{i}$, together with the argument variable $a_{i}$ to constitute the OWA pair $\left\langle u_{i}, a_{i}\right\rangle, i=1, \cdots, n$. The latter was introduced by Yager and Filev [27] as the induced OWA (IOWA) operator. When compared with the OWA operator [30], the reordering step of the IOWA operator 
is determined by the order inducing variable rather than by the argument variable itself. Therefore, it can be widely and conveniently applied to some classic situations, such as the Nearest-Neighbor model [27] and the best yesterday model [27], etc. Its definition is as follows.

Definition 1 ([27]). Let $\mathbb{R}$ be the set of all real numbers and $\Omega$ be the set that is equipped with a linear order. An IOWA operator of dimension $n$ is a mapping IOWA $:(\Omega \times \mathbb{R})^{n} \longrightarrow \mathbb{R}$, which has an associated weighting vector $W=\left(\omega_{1}, \cdots, \omega_{n}\right)$ with $\omega_{i} \in[0,1]$ and $\sum_{i=1}^{n} \omega_{j}=1, i=$ $1,2, \cdots, n$, in order to aggregate the arguments $a_{i}$ of the OWA pairs $\left\langle u_{i}, a_{i}\right\rangle, i=1,2, \cdots, n$ that reorder by the order inducing variable $u_{i}$, according to the following formula:

$$
\operatorname{IOWA}\left(\left\langle u_{1}, a_{1}\right\rangle, \cdots,\left\langle u_{n}, a_{n}\right\rangle\right)=\sum_{i=1}^{n} \omega_{i} a_{u-i n d e x(i)}
$$

where $u$-index $(\cdot)$ is an index function, such that $u$-index $(i)$ is the index of the argument variable, which is paired with the $i^{\text {th }}$ largest element of the set $\left\{u_{1}, \cdots, u_{n}\right\}$.

Example 1. Assume a set of OWA pairs $\{\langle 7,0.4\rangle,\langle 9,0.3\rangle,\langle 4,0.7\rangle\}$ has been assigned a weighting vector $\{0.6,0.1,0.3\}$, then the notation of reordered OWA pairs is $\{\langle 9,0.3\rangle,\langle 7,0.4\rangle,\langle 4,0.7\rangle\}$, and, with the application of the Equation (2), we obtain the results $\operatorname{IOWA}\left(\left\langle u_{1}, a_{1}\right\rangle,\left\langle u_{2}, a_{2}\right\rangle,\left\langle u_{3}, a_{3}\right\rangle\right)=$ $0.3 \times 0.6+0.4 \times 0.1+0.7 \times 0.3=0.43$. If we apply arguments $\{0.4,0.3,0.7\}$ with the OWA operator [30], we obtain the results $\mathbf{O W A}\left(a_{1}, a_{2}, a_{3}\right)=0.7 \times 0.6+0.4 \times 0.1+0.3 \times 0.3=0.55$.

Therefore, the essential difference between the OWA operator and IOWA operator is the reordering step. The IOWA operator is based on the order inducing variable, while the OWA operator is based on the argument variable itself.

\subsection{Fuzzy Linguistic Quantifiers: Computing Weights for Owa Operators}

Among the multiple choices for choosing OWA weights, Yager $[24,28,30]$ introduced the fuzzy linguistic quantifier $Q$, which guides the OWA operator in order to compute the weight of the OWA operator. In the simplest form, computing weights for the OWA operator on a basic unit-interval monotonic (BUM) function [28] $f:[0,1] \rightarrow[0,1]$, which is characterized as: (i) $f(0)=0$; (ii) $f(1)=1$; (iii) $f(x) \leq f(y)$, if $0 \leq x<y \leq 1$. However, we often use the BUM function $Q(r), \forall r \in[0,1]$, which is characterized by two parameters $\alpha, \beta \in[0,1]$ as:

$$
Q(r)=\left\{\begin{array}{c}
0, \quad \text { if } 0 \leq r<\alpha \\
\frac{r-\alpha}{\beta-\alpha}, \quad \text { if } \alpha \leq r \leq \beta \\
1, \quad \text { if } \beta<r \leq 1
\end{array}\right.
$$

Several examples of instantiations of $\alpha$ and $\beta$ for linguistic quantifiers are as follows:

(1) If $\alpha=\beta=0$, then BUM function $Q$ represents the linguistic quantifier "for all";

(2) If $\alpha=\beta=1$, then BUM function $Q$ represents the linguistic quantifier "there exist";

(3) If $\alpha=0, \beta=1$, then BUM function $Q$ represents the linguistic quantifier "mean";

(4) If $\alpha=0.3, \beta=0.8$, then BUM function $Q$ represents the linguistic quantifier "most";

(5) If $\alpha=0, \beta=0.5$, then BUM function $Q$ represents the linguistic quantifier "at least half"; and,

(6) If $\alpha=0.5, \beta=0.8$, then BUM function $Q$ represents the linguistic quantifier "as many as possible".

Therefore, the weight $\omega_{i}$ for the OWA operator is computed as

$$
\omega_{i}=Q\left(\frac{i}{n}\right)-Q\left(\frac{i-1}{n}\right), i=1, \ldots, n
$$


There is an extension of it, the so-called importance weighted quantifier guided aggregation [28], which adds an additional the importance variable $\alpha_{i}$ in order to form a 2-tuple noted as $\left\langle a_{i}, \alpha_{i}\right\rangle, i=1, \cdots, n$, so that we define the OWA weight and OWA operator as follows:

$$
\begin{gathered}
\omega_{i}=Q\left(\frac{I(i)}{I(n)}\right)-Q\left(\frac{I(i-1)}{I(n)}\right) \\
\operatorname{OWA}\left(\left\langle a_{1}, \alpha_{1}\right\rangle, \ldots,\left\langle a_{n}, \alpha_{n}\right\rangle\right)=\sum_{i=1}^{n} \omega_{i} a_{a-i n d e x(i)}
\end{gathered}
$$

where $I(i)=\sum_{k=1}^{i} \alpha_{a-i n d e x}(k), i, k=1, \cdots, n$ with $a-i n d e x(\cdot)$ is an index function, such that $a-\operatorname{index}(i)$ is the index of the important variable, which is paired with the $i^{\text {th }}$ largest element of the set $\left\{a_{1}, \cdots, a_{n}\right\}$.

Example 2. Let a BUM function $Q$ with $\alpha=0, \beta=0.5$ that represents the linguistic quantifier "at least half" and the aggregated 2-tuple is $\{\langle 0.5,0.6\rangle,\langle 1,0.3\rangle,\langle 0.4,0.5\rangle\}$, then the ordered 2-tuple is $(\langle\mathbf{1}, 0.3\rangle,\langle\mathbf{0 . 5}, 0.6\rangle,\langle\mathbf{0 . 4}, 0.5\rangle)$ and $I(1)=0.3, I(2)=0.9, I(3)=2.4$.

We will obtain the OWA weight

$$
\begin{aligned}
\omega_{1}=Q\left(\frac{0.3}{2.4}\right)-Q\left(\frac{0}{2.4}\right) & =0.25, \omega_{2}=Q\left(\frac{0.9}{2.4}\right)-Q\left(\frac{0.3}{2.4}\right)=0.5, \omega_{3}=Q\left(\frac{2.4}{2.4}\right)-Q\left(\frac{0.9}{2.4}\right)=0.25 \\
\text { and } & \\
& \text { OWA }(\langle 0.5,0.6\rangle,\langle 1,0.3\rangle,\langle 0.4,0.5\rangle)=\mathbf{1} \times 0.25+\mathbf{0 . 5} \times 0.5+\mathbf{0 . 4} \times 0.25=1.5 .
\end{aligned}
$$

\subsection{Elicit Information}

Recently, Labella et al. [15] proposed a new linguistic representation model, the socalled Extended Comparative Linguistic Expressions with Symbolic Translation (ELICIT)

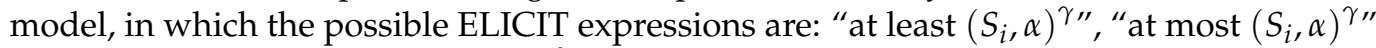

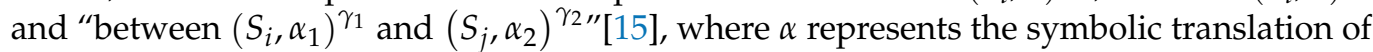
the 2-tuple represented model $\left(s_{i}, \alpha\right)[9,16]$ and $\gamma$ is an additional "adjustment" parameter that provides essential information within the computation process to the trapezoidal fuzzy numbers. Noticeably, if $\gamma=\alpha=0$, then the ELICIT expressions reduce to the CLEs $[2,17,33]$, for instance, "at least $S_{i}$ ", "at most $S_{i}$ ", and "between $S_{i}$ and $S_{j}$ ". Therefore, the ELICIT model is an extension of hesitant fuzzy linguistic term sets (HFLTS) $[2,17]$ based on the continuous domain of comparative linguistic expressions (CLEs).

The ELICIT model extends the computing with words (CW) process to the ELICIT-CW approach [15] comprises three parts in turn: the translation process, the manipulation process and the re-translation process. In the ELICIT-CW scheme, the translation process via functions $\zeta^{-1}$ in order to transform the input ELICIT information into trapezoidal fuzzy numbers, so that the manipulation process could fuse trapezoidal fuzzy numbers utilizing the proposed aggregation operator and also help to accurately complete the $\mathrm{CW}$ process; finally, the re-translation process through $\zeta$ translates the aggregated result to the ELICIT expression that is easy to understand; more details are defined as follows:

Definition 2 ([15]). Let $x$ be an ELICIT expression and $T(a, b, c, d)$ be a trapezoidal fuzzy number. The function $\zeta^{-1}$ transforms the ELICIT expression into a trapezoidal fuzzy number, as

$$
\zeta^{-1}: x \longmapsto T(a, b, c, d)
$$

Such that, for an input ELICIT expression, it is transformed into an equivalent trapezoidal fuzzy number. Further details regarding the ELICIT expressions "at least $\left(S_{i}, \alpha\right)^{\gamma \text { ", }}$

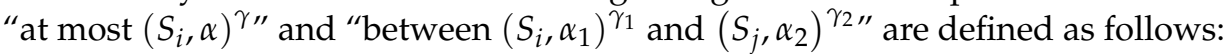

Definition 3 ([15]). Let $\mathcal{F}$ be the set of all possible ELICIT expressions and $S=\left\{S_{0}, S_{1}, \cdots, S_{g}\right\}$ be a set of linguistic terms, then $\forall x \in \mathcal{F}$, the function $\zeta^{-1}(x)$ is given by 
(1) if $x=$ at least $\left(S_{i}, \alpha\right)^{\gamma}$ and $T_{\text {ELICIT }}\left(a^{\prime}, b^{\prime}, 1,1\right)$ is the fuzzy envelope of at least $\left(S_{i}, \alpha\right)^{\gamma}$, then

$$
\begin{aligned}
\zeta^{-1}(x) & =T(a, b, 1,1) \\
a & =a^{\prime}+\gamma \\
b & =b^{\prime} ;
\end{aligned}
$$

(2) if $x=$ at most $\left(S_{i}, \alpha\right)^{\gamma}$ and $T_{\text {ELICIT }}\left(0,0, c^{\prime}, d^{\prime}\right)$ is the fuzzy envelope of at least $\left(S_{i}, \alpha\right)^{\gamma}$, then

$$
\begin{aligned}
\zeta^{-1}(x) & =T(0,0, c, d) \\
c & =c^{\prime} \\
d & =d^{\prime}+\gamma
\end{aligned}
$$

(3) if $x=$ between $\left(S_{i}, \alpha_{1}\right)^{\gamma_{1}}$ and $\left(S_{j}, \alpha_{2}\right)^{\gamma_{2}}$ and $T_{\text {ELICIT }}\left(a^{\prime}, b^{\prime}, c^{\prime}, d^{\prime}\right)$ is the fuzzy envelope of between $\left(S_{i}, \alpha_{1}\right)^{\gamma_{1}}$ and $\left(S_{j}, \alpha_{2}\right)^{\gamma_{2}}$, then

$$
\begin{aligned}
\zeta^{-1}(x) & =T(a, b, c, d) \\
a & =a^{\prime}+\gamma_{1} \\
b & =b^{\prime} \\
c & =c^{\prime} \\
d & =d^{\prime}+\gamma_{2}
\end{aligned}
$$

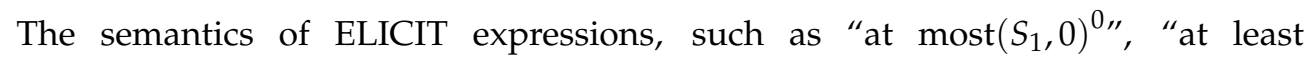

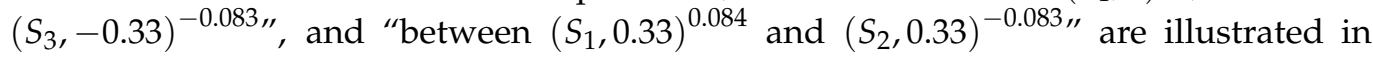
Figure 1, as follows:

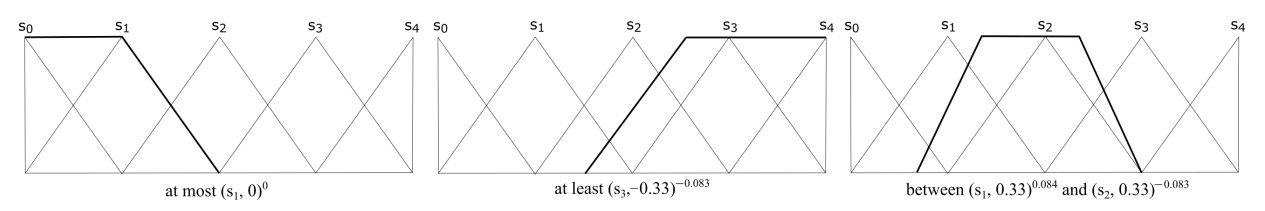

Figure 1. ELICIT expression examples.

The inverse function of $\zeta^{-1}$ is noted as $\zeta$ that is a mapping $\zeta: \widetilde{\beta} \longmapsto x$ that re-translates the trapezoidal fuzzy number $\widetilde{\beta}$ into an ELICIT expression.

Definition 4 ([15]). Let $S=\left\{S_{0}, S_{1}, \cdots, S_{g}\right\}$ be a set of linguistic terms and $\widetilde{\beta}$ a trapezoidal fuzzy number. The function $\zeta$ is given by

$$
\zeta(\widetilde{\beta})=\left\{\begin{array}{l}
\text { at least }\left(S_{i}, \alpha\right)^{\gamma}, \quad \text { if } \widetilde{\beta}=T(a, b, 1,1) \\
\text { at most }\left(S_{i}, \alpha\right)^{\gamma}, \text { if } \widetilde{\beta}=T(0,0, c, d) \\
\text { between }\left(S_{i}, \alpha_{1}\right)^{\gamma_{1}} \text { and }\left(S_{j}, \alpha_{2}\right)^{\gamma_{2}}, \text { if } \widetilde{\beta}=T(a, b, c, d)
\end{array}\right.
$$

As mentioned above, in essence, the fusion CLEs or ELICIT expressions is the fusion of trapezoidal fuzzy numbers during the ELICIT-CW approach. Therefore, we should apply classical operators on them, such as the OWA operator, the IOWA operator, and other canonical operators, or expand them with crisp or fuzzy weights. Uncertain or fuzzy environments (that are represented by fuzzy numbers in most cases) often occur in the daily life of decision makers. Therefore, if we want to manage it with crisp weights, the process is similar, but, when comparing it with the combination of fuzzy weights, it is difficult to complete the calculation process, because the general mathematical multiplication and division of trapezoidal fuzzy numbers will no longer retain the trapezoid [34], which is not conducive to completing the ELICIT-CW approach. So far, there are only the fuzzy arithmetic mean operator proposed by Labella et al. [15] and the Bonferroni mean operator 
applied by Dutta et al. [21] for aggregating the ELICIT information. Therefore, the aggregation operators of ELICIT information, especially the information with fuzzy weight, is worthy of our study.

\section{Iowa Operators for Aggregating Elicit Information}

In the introduction, it has been stated the importance of the aggregation process for decision making processes and how a generic aggregation framework could be applicable to a wider set of decision making contexts through the modeling and interpretation of the attached parameters of the aggregation model, as per the demand of the decision context. In this section, we propose extending the IOWA operator in order to deal with ELICIT information. However, we do not aim to make a simple and straightforward extension of the classical IOWA operator; we intend to develop suitable and necessary models of the IOWA operator for dealing with ELICIT information in decision making. Therefore, our proposal consists of defining the following operators and the order inducing variable:

- $\quad$ ELICIT-IOWA operator: this operator extends the IOWA operator to aggregate ELICIT arguments with crisps weights. It will also describe the way to compute the crisp weights for this operator.

- $\quad$ ELICIT-t1-IOWA operator: this operator extends the IOWA operator to aggregate ELICIT arguments with fuzzy weights. How to compute these fuzzy weights for this operator will be described.

- The ELICIT order inducing variable: a brief study will also be described in order to investigate the use of ELICIT variables as the order inducing variable in previous operators.

\subsection{Elicit-Iowa Operator}

Here, we will replace the argument variable $a_{i}$ of the OWA pair $\left\langle u_{i}, a_{i}\right\rangle$ [27] with ELICIT expression $x_{i}$, and then the aggregation of the new OWA pairs $\left\langle u_{i}, x_{i}\right\rangle, i=1, \cdots, n$ can be defined as follows:

Definition 5. Let $\mathcal{F}$ be the set of all possible ELICIT expressions and $\Omega$ be the set that is equipped with a linear order. An ELICIT-IOWA operator of dimension $n$ is a mapping ELICIT - IOWA : $(\Omega \times \mathcal{F})^{n} \longrightarrow \mathcal{F}$ that has an associated weighting vector $W=\left\{\omega_{i}\right\}_{i=1}^{n}$ of dimension $n$ with $\omega_{i} \in[0,1], i=1,2, \cdots, n$ and $\sum_{i=1}^{n} \omega_{i}=1$, then

$$
\begin{aligned}
\operatorname{ELICIT}-\operatorname{IOWA}\left(\left\langle u_{1}, x_{1}\right\rangle, \cdots,\left\langle u_{n}, x_{n}\right\rangle\right) & =\zeta\left(\sum_{i=1}^{n} \omega_{i} \times \zeta^{-1}\left(x_{u-i n d e x(i)}\right)\right) \\
& =\zeta\left(\sum_{i=1}^{n} \omega_{i} \times T\left(a_{i}, b_{i}, c_{i}, d_{i}\right)\right) \\
& =\zeta\left(T\left(\sum_{i=1}^{n} \omega_{i} a_{i}, \sum_{i=1}^{n} \omega_{i} b_{i}, \sum_{i=1}^{n} \omega_{i} c_{i}, \sum_{i=1}^{n} \omega_{i} d_{i}\right)\right)
\end{aligned}
$$

where $\times$ represents the multiplication between the scalar and fuzzy numbers. Let $u-i n d e x(\cdot)$ be an index function, such that $u$-index $(i)$ is the index of the argument variable (see Definition 1 and Example 1), which is paired with the $i^{\text {th }}$ largest element of the set $\left\{u_{1}, \cdots, u_{n}\right\}$. $\zeta^{-1}\left(x_{u-\text { index }(i)}\right)=T\left(a_{i}, b_{i}, c_{i}, d_{i}\right), i=1,2, \cdots, n$ is the equivalent trapezoidal fuzzy number corresponding to the ELICIT expression $x_{u-i n d e x(i)}$ and $\zeta$ is the inverse function of $\zeta^{-1}$.

The ELICIT-IOWA operator is also the OWA based operator, so that the weight can be obtained from a basic unit-interval monotonic (BUM) function [28] $Q:[0,1] \longrightarrow[0,1]$, 
which is characterized as: (i) $Q(0)=0$; (ii) $Q(1)=1$; (iii) $Q(x) \leq Q(y)$, if $0 \leq x<y \leq 1$; then, the weight

$$
\omega_{i}=Q\left(\frac{i}{n}\right)-Q\left(\frac{i-1}{n}\right), i=1, \ldots, n
$$

In the IOWA situation, there is a straight forward extension of the OWA pair $\left\langle u_{i}, x_{i}\right\rangle$ with an additional variable, the so-called weight variable $\alpha_{i} \in[0,1][35]$ that is the associated weight of each argument $x_{i}$, noted as a triple $\left\langle u_{i}, x_{i}, \alpha_{i}\right\rangle, i=1, \cdots, n$. The weight variable $\alpha_{i}$ helps to obtain the crisp weight that is attached to the ordered position of the ELICIT-IOWA operator, which is called the functional generated weight [35], and this crisp weight $\omega_{i}$ can be computed as follows:

$$
\omega_{i}=Q\left(\frac{I(i)}{I(n)}\right)-Q\left(\frac{I(i-1)}{I(n)}\right), i=1, \ldots, n
$$

where $I(i)=\sum_{k=1}^{i} \alpha_{u-i n d e x}(k), k=1, \cdots, n$. Additionally, let $u-i n d e x(\cdot)$ be an index function, such that $u$-index $(i)$ is the index of the argument variable $x_{j}$ and the weight variable $\alpha_{j}$, which are paired with the $i^{\text {th }}$ largest element of the set $\left\{u_{1}, \cdots, u_{n}\right\}$.

Nevertheless, if the weight variable $\alpha_{j}$ is added to OWA pairs $\left\{\left\langle u_{i}, x_{i}\right\rangle\right\}_{i=1}^{n}$ of the ELICIT-IOWA operator to obtain triples $\left\{\left\langle u_{i}, x_{i}, \alpha_{i}\right\rangle\right\}_{i=1}^{n}$, and the BUM function $Q$ is used, then we will obtain the definition of the important ELICIT-IOWA (ELICIT-I-IOWA) operator. That is:

Definition 6. Let $\mathcal{F}$ be the set of all possible ELICIT expressions and $\Omega$ be the set that is equipped with a linear order. An ELICIT-I-IOWA operator is a mapping ELICIT - I - IOWA : $(\Omega \times \mathcal{F} \times[0,1])^{n} \longrightarrow \mathcal{F}$ that has an associated weighting vector $W=\left\{\omega_{i}\right\}_{i=1}^{n}$ of dimension $n$ with $\omega_{i} \in[0,1], i=1,2, \cdots, n$ and $\sum_{i=1}^{n} \omega_{i}=1$ computed by the BUM function $Q$ and the ELICIT-I-IOWA operator is defined as follows:

$$
\begin{aligned}
\operatorname{ELICIT}-\mathbf{I}-\operatorname{IOWA}\left(\left\langle u_{1}, x_{1}, \alpha_{1}\right\rangle, \ldots,\left\langle u_{n}, x_{n}, \alpha_{n}\right\rangle\right) & =\zeta\left(\sum_{i=1}^{n} \omega_{i} \times \zeta^{-1}\left(x_{u-\text { index }(i)}\right)\right) \\
& =\zeta\left(\sum_{i=1}^{n} \omega_{i} \times T\left(a_{i}, b_{i}, c_{i}, d_{i}\right)\right) \\
& =\zeta\left(T\left(\sum_{i=1}^{n} \omega_{i} a_{i}, \sum_{i=1}^{n} \omega_{i} b_{i}, \sum_{i=1}^{n} \omega_{i} c_{i}, \sum_{i=1}^{n} \omega_{i} d_{i}\right)\right)
\end{aligned}
$$

where $\omega_{i}=Q\left(\frac{I(i)}{I(n)}\right)-Q\left(\frac{I(i-1)}{I(n)}\right)$ within $I(i)=\sum_{k=1}^{i} \alpha_{u-i n d e x(k)}, i, k=1, \cdots, n$. Additionally, $\times$ represents the multiplication between scalar and fuzzy numbers. Let $u-$ index $(\cdot)$ be an index function such that $u-i n d e x(i)$ is the index of the argument variable and the weight variable, which is paired with the $i^{\text {th }}$ largest element of the set $\left\{u_{1}, \cdots, u_{n}\right\} . \zeta^{-1}\left(x_{u-\text { index }(i)}\right)=T\left(a_{i}, b_{i}, c_{i}, d_{i}\right)$, $i=1,2, \cdots, n$ is the equivalent trapezoidal fuzzy number corresponding to the ELICIT expression $x_{u-\text { index }(i)}$ and $\zeta$ is the inverse function of $\zeta^{-1}$.

Noticeably, if we apply the ELICIT-I-IOWA operator with the BUM function $Q(r)=r$, then we will obtain the functional weight as:

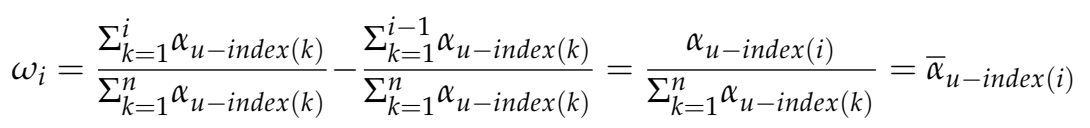

and

$$
\begin{aligned}
\text { and } & \\
\text { ELICIT - I - IOWA }\left(\left\langle u_{1}, x_{1}, \alpha_{1}\right\rangle_{,}, \cdots,\left\langle u_{n}, x_{n}, \alpha_{n}\right\rangle\right) & =\zeta\left(\sum_{i=1}^{n} \bar{\alpha}_{u-i n d e x(i)} \times \zeta^{-1}\left(x_{u-\operatorname{index}(i)}\right)\right) \\
& =\zeta\left(\sum_{i=1}^{n} \bar{\alpha}_{i} \times \zeta^{-1}\left(x_{i}\right)\right)
\end{aligned}
$$


hence, it is the weighted average (WA) operator for ELICIT information.

Remark 1. Taking into account the fuzzy induced quasi-arithmetic OWA (QFIOWA) operator [36], which is a mapping QFIOWA : $(\Omega \times \Psi)^{n} \longrightarrow \Psi$ that has associated weights $\omega_{i} \in[0,1], i=1, \cdots, n$, such that $\Sigma_{i=1}^{n} \omega_{i}=1$ and

$$
\operatorname{QFIOWA}\left(\left\langle u_{1}, \hat{a}_{1}\right\rangle, \cdots,\left\langle u_{n}, \hat{a}_{n}\right\rangle\right)=g^{-1}\left(\sum_{i=1}^{n} \omega_{i} \times g\left(\hat{a}_{u-i n d e x(i)}\right)\right)
$$

where $\Omega$ is the set equipped with a linear order and $\Psi$ is the set of all fuzzy numbers. $g$ is a strictly continuous monotonic function.

If $g(x)=x^{\lambda}(\lambda \neq 0)$, then the QFIOWA is the fuzzy induced generalized OWA (FIGOWA) operator, defined as

$$
\operatorname{FIGOWA}\left(\left\langle u_{1}, \hat{a}_{1}\right\rangle, \cdots,\left\langle u_{n}, \hat{a}_{n}\right\rangle\right)=\left(\sum_{i=1}^{n} \omega_{i}\left(\hat{a}_{u-i n d e x}(i)\right)^{\lambda}\right)^{\lambda^{-1}} .
$$

In a nutshell, because the ELICIT expression is equivalent to a trapezoidal fuzzy number through the function $\zeta^{-1}$, i.e., $\zeta^{-1}(\mathcal{F}) \subseteq \Psi$, where $\mathcal{F}$ is the set of all possible ELICIT expressions and $\Psi$ is the set of all fuzzy numbers, if we add a strictly continuous monotone function $g$ to the ELICIT-IOWA operator, the quasi-ELICIT-IOWA (ELICIT-QIOWA) operator will be defined as follows:

$$
\text { ELICIT - QIOWA }\left(\left\langle u_{1}, x_{1}\right\rangle, \cdots,\left\langle u_{n}, x_{n}\right\rangle\right)=\zeta\left(g^{-1}\left(\sum_{i=1}^{n} \omega_{i} \times g\left(\zeta^{-1}\left(x_{u-i n d e x}(i)\right)\right)\right)\right)
$$

Furthermore, let $g$ be an exponential function where $g(x)=x^{\lambda}$ with parameter $\lambda \neq 0$, then the quasi-ELICIT-IOWA operator is the generalized ELICIT-IOWA (ELICIT-GIOWA) operator and

$$
\operatorname{ELICIT}-\operatorname{GIOWA}\left(\left\langle u_{1}, x_{1}\right\rangle, \cdots,\left\langle u_{n}, x_{n}\right\rangle\right)=\zeta\left(\left(\sum_{i=1}^{n} \omega_{i} \times\left(\zeta^{-1}\left(x_{u-i n d e x(i)}\right)\right)^{\lambda}\right)^{\lambda^{-1}}\right)
$$

Several special cases, depending on the various values of the parameter $\lambda$, are as follows:

(1) If $\lambda=1$, then the ELICIT-GIOWA operator reduces to the ELICIT-IOWA operator;

(2) If $\lambda \rightarrow 0$, then

$$
\operatorname{ELICIT}-\operatorname{GIOWA}\left(\left\langle u_{1}, x_{1}\right\rangle, \cdots,\left\langle u_{n}, x_{n}\right\rangle\right)=\zeta\left(\sum_{i=1}^{n}\left(\zeta^{-1}\left(x_{u-i n d e x(i)}\right)\right)^{\omega_{i}}\right)
$$

(3) If $\lambda=-1$, then the ELICIT-GIOWA operator is close to the Harmonic average $H=\frac{n}{\sum_{i=1}^{n} \frac{1}{x_{i}}}$ as

$$
\text { ELICIT - GIOWA } \left.\left(\left\langle u_{1}, x_{1}\right\rangle, \cdots,\left\langle u_{n}, x_{n}\right\rangle\right)=\zeta\left(\frac{\prod_{i=1}^{n} \zeta^{-1}\left(x_{u-\text { index }(i)}\right)}{\sum_{i=1}^{n} \omega_{i}\left(\prod_{\substack{n=1 \\ j \neq i}}^{n} \zeta^{-1}\left(x_{u \text {-index }(j)}\right)\right.}\right)\right)
$$

(4) If $\lambda \rightarrow+\infty$, then

$$
\text { ELICIT - GIOWA }\left(\left\langle u_{1}, x_{1}\right\rangle, \cdots,\left\langle u_{n}, x_{n}\right\rangle\right) \rightarrow \max _{i}\left\{x_{i}\right\}
$$

(5) If $\lambda \rightarrow-\infty$, then

$$
\text { ELICIT - GIOWA }\left(\left\langle u_{1}, x_{1}\right\rangle, \cdots,\left\langle u_{n}, x_{n}\right\rangle\right) \rightarrow \min _{i}\left\{x_{i}\right\}
$$


Remark 2. Some special cases of the ELICIT-IOWA operator by using different crisp weighting vectors $W$ are briefly detailed.

(1) If $W=W^{*}=\{1,0, \cdots, 0\}$, then

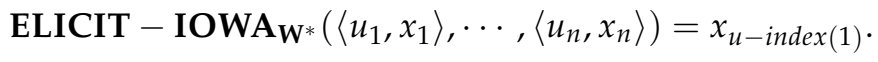

(2) If $W=W_{*}=\{0, \cdots, 0,1\}$, then

$$
\text { ELICIT - } \text { IOWA }_{\mathbf{W}_{*}}\left(\left\langle u_{1}, x_{1}\right\rangle, \cdots,\left\langle u_{n}, x_{n}\right\rangle\right)=x_{u-i n d e x}(n)
$$

(3) If $W=W_{N}=\left\{\frac{1}{n}, \cdots, \frac{1}{n}\right\}$, then

$$
\text { ELICIT }- \text { IOWA }_{\mathbf{W}_{\mathbf{N}}}\left(\left\langle u_{1}, x_{1}\right\rangle, \cdots,\left\langle u_{n}, x_{n}\right\rangle\right)=\zeta\left(T\left(\frac{\sum_{i=1}^{n} a_{i}}{n}, \frac{\sum_{i=1}^{n} b_{i}}{n}, \frac{\sum_{i=1}^{n} c_{i}}{n}, \frac{\sum_{i=1}^{n} d_{i}}{n}\right)\right)
$$

It is the same as the fuzzy arithmetic mean operator that was introduced in [15];

\subsection{Elicit-T1-Iowa Operator}

Inspired by the type-1 OWA operator, which extends the OWA operator [30] to the type-1 fuzzy sets on the basis of Zadeh's extension principle [12-14]. We will then follow the definition of the type-1 OWA operator that is based on the IOWA operator [27] in order to obtain the induced type-1 OWA (t1-IOWA) operator as follows:

Definition 7. Let $F([0,1])$ be the set of all type-1 fuzzy sets defined on the universe of discourse $[0,1]$ and $\Omega$ be the set that is equipped with a linear order. An t1-IOWA operator of dimension $n$ is a mapping

$$
\begin{gathered}
\Phi_{\text {t1-IOWA }}:(\Omega \times F([0,1]))^{n} \longrightarrow F([0,1]) \\
\left(\left\langle u_{1}, A_{1}\right\rangle, \cdots,\left\langle u_{n}, A_{n}\right\rangle\right) \mapsto A
\end{gathered}
$$

that has an associated weighting vector $\hat{W}=\left\{\hat{w}_{i}\right\}_{i=1}^{n}$ of dimension $n$ with fuzzy weights $\hat{w}_{i} \in$ $F([0,1]), i=1,2, \cdots, n$, such that

$$
\begin{aligned}
& \Phi_{\mathbf{t 1}-\mathbf{I O W A}}\left(\left\langle u_{1}, A_{1}\right\rangle, \cdots,\left\langle u_{n}, A_{n}\right\rangle\right)=A=\frac{\sum_{i=1}^{n} \hat{w}_{i} \otimes A_{u-\text { index }(i)}}{\sum_{i=1}^{n} \hat{w}_{i}} \\
& A=\left\{\left(y, \mu_{A}(y)\right) \mid y=\frac{\sum_{i=1}^{n} \omega_{i} y_{i}}{\sum_{i=1}^{n} \omega_{i}}, \forall \omega_{i}, y_{i} \in[0,1]\right\} \\
& \mu_{A}(y)=\sup \quad \begin{array}{c}
y=\frac{\sum_{i=1}^{n} \omega_{i} y_{i}}{\sum_{i=1}^{n} \omega_{i}} \\
\forall \omega_{i}, y_{i} \in[0,1]
\end{array}\left\{\mu_{\hat{w}_{1}}\left(\omega_{1}\right) * \mu_{A_{u-i n d e x}(1)}\left(y_{1}\right) * \cdots * \mu_{\hat{w}_{n}}\left(\omega_{n}\right) * \mu_{A_{u-i n d e x}(n)}\left(y_{n}\right)\right\}
\end{aligned}
$$

where $\otimes$ is the multiplication operation on fuzzy numbers and $*$ is a t-norm operator. Let $u-$ index $(\cdot)$ be an index function, such that $u-i n d e x(i)$ is the index of the argument variable, which is paired with the $i^{\text {th }}$ largest element of the set $\left\{u_{1}, \cdots, u_{n}\right\}$.

According to the predefined, if the t1-IOWA operator has an associated interval weight $\widetilde{w}_{i} \subseteq[0,1]$; in other words, the membership of the interval weight equals 1 , i.e., $\mu_{\widetilde{w}_{i}}\left(\omega_{i}\right)=1, \forall \omega_{i} \in \widetilde{w}_{i}, i=1,2, \cdots, n$, we will easily obtain this formula 


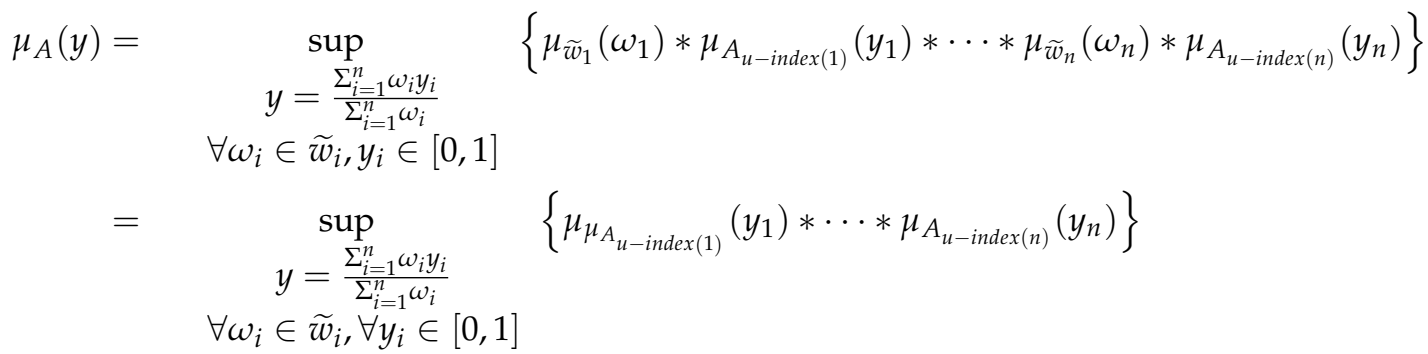

Therefore, the previous definition can be shown as follows:

Definition 8. An t1-IOWA operator of dimension $n$ is a mapping $\Phi_{\mathbf{t} 1-\mathbf{I O W A}}:(\Omega \times F([0,1]))^{n}$ $\longrightarrow F([0,1])$ that has an associated weighting vector $\widetilde{W}=\left\{\widetilde{w}_{i}\right\}_{i=1}^{n}$ of dimension $n$ with interval weights $\widetilde{w}_{i} \subseteq[0,1], i=1,2, \cdots, n$ and $\mu_{\widetilde{w}_{i}}\left(\omega_{i}\right)=1, \forall \omega_{i} \in \widetilde{w}_{i}$, such that

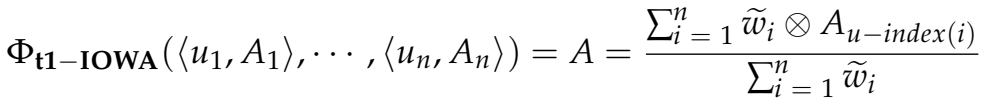

$$
\begin{aligned}
& A=\left\{\left(y, \mu_{A}(y)\right) \mid y=\frac{\sum_{i=1}^{n} \omega_{i} y_{i}}{\sum_{i=1}^{n} \omega_{i}}, \forall \omega_{i} \in \widetilde{w}_{i}, \forall y_{i} \in[0,1]\right\}
\end{aligned}
$$

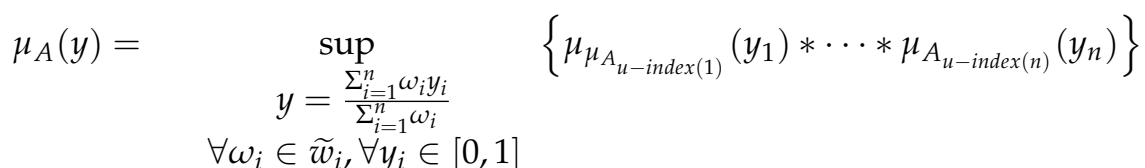

where $\otimes$ is multiplication operation on fuzzy numbers and $*$ is a t-norm operator. Let $u-i n d e x(\cdot)$ be an index function, such that $u$-index $(i)$ is the index of the argument variable, which is paired with the $i^{\text {th }}$ largest element of the set $\left\{u_{1}, \cdots, u_{n}\right\}$.

It is very complicated to calculate with the extended principle, because we need to discretize the domain of the fuzzy set. Therefore, we rely on $\alpha$-cut based method to solve this problem, which is more computationally efficient and provide better approximations. To do this, we will follow the concept of the $\alpha$ - level type-1 OWA operator [37] guided by $\alpha$-cut of interval weights to obtain the definition of the $\alpha$ - level t1-IOWA operator with fuzzy weights as follows:

Definition 9. Let $\left\{A_{i}^{\alpha}\right\}_{i=1}^{n}$ represents the $\alpha$-cut of fuzzy sets $\left\{A_{i}\right\}_{i=1}^{n}$, and then denote the corresponding OWA pairs as $\left\{\left\langle u_{i}, A_{i}^{\alpha}\right\rangle\right\}_{i=1}^{n}$. Let $\left\{\hat{w} \hat{w}_{i}^{\alpha}\right\}_{i=1}^{n}$ represent the $\alpha-$ cut of fuzzy weights $\left\{\hat{w}_{i}\right\}_{i=1}^{n}$. For each $\alpha \in[0,1]$, an $\alpha$ - level t1-IOWA operator is defined as $\boldsymbol{\Phi}_{\mathbf{t} 1 \text {-IOWA }}^{\alpha}:\left(\left\langle u_{1}, A_{1}^{\alpha}\right\rangle, \cdots,\left\langle u_{n}, A_{n}^{\alpha}\right\rangle\right)$ $\mapsto A^{\alpha}$, such that

$$
\begin{gathered}
A^{\alpha}=\left\{\frac{\sum_{i=1}^{n} \omega_{i} y_{i}}{\sum_{i=1}^{n} \omega_{i}} \mid \forall \omega_{i} \in \hat{w}_{i}^{\alpha}, \forall y_{i} \in A_{u-i n d e x(i)}^{\alpha}\right\} \\
A=\bigcup_{0 \leq \alpha \leq 1} \alpha A^{\alpha} \\
\mu_{A}(y)=\bigvee_{\alpha: y \in A^{\alpha}} \alpha
\end{gathered}
$$

where $\hat{w}_{i}^{\alpha}=\left\{\omega \in[0,1] \mid \mu_{\hat{w}_{i}}(\omega) \geq \alpha\right\}$ and $A_{u-\text { index }(i)}^{\alpha}=\left\{y \in[0,1] \mid \mu_{A_{u-\text { index }(i)}}(y) \geq \alpha\right\}$. Let $u$-index (.) be an index function, such that $u-i n d e x(i)$ is the index of the argument variable, which is paired with the $i^{\text {th }}$ largest element of the set $\left\{u_{1}, \cdots, u_{n}\right\}$.

The proposed t1-IOWA operator provides a basic IOWA aggregation framework for aggregating fuzzy information. Because each ELICIT expression is equivalent to a fuzzy number, we can define the aggregation of ELICIT expressions based on the t1-IOWA 
operator framework that we proposed. For this purpose, we replace the fuzzy parameter variable with the ELICIT expression. Therefore, we will extend the t1-IOWA operator in order to aggregate ELICIT information, where the fuzzy weight is induced by the type-2 linguistic quantifier [32].

Definition 10. Let $\mathcal{F}$ be the set of all possible ELICIT expressions and $\Omega$ be the set that is equipped with a linear order. An ELICIT-t1-IOWA operator of dimension $n$ is a mapping ELICIT - t1 - IOWA : $(\Omega \times \mathcal{F})^{n} \longrightarrow \mathcal{F}$ that has an associated weighting vector $\hat{W}=\left\{\hat{w}_{i}\right\}_{i=1}^{n}$ of dimension $n$ with fuzzy weights $\hat{w}_{i} \in F([0,1]), i=1,2, \cdots, n$, such that

$$
\text { ELICIT }-\mathbf{t 1}-\operatorname{IOWA}\left(\left\langle u_{1}, x_{1}\right\rangle, \cdots,\left\langle u_{n}, x_{n}\right\rangle\right)=\zeta\left(\frac{\sum_{i=1}^{n} \hat{w}_{i} \otimes \zeta^{-1}\left(x_{u-\operatorname{index}(i)}\right)}{\sum_{i=1}^{n} \hat{w}_{i}}\right)
$$

where $\otimes$ is the multiplication operation on fuzzy numbers. Let $u-i n d e x(\cdot)$ be an index function, such that $u$-index $(i)$ is the index of the argument variable, which is paired with the $i^{\text {th }}$ largest element of the set $\left\{u_{1}, \cdots, u_{n}\right\} . \zeta^{-1}\left(x_{u-i n d e x(i)}\right)=T\left(a_{i}, b_{i}, c_{i}, d_{i}\right), i=1,2, \cdots, n$ is the equivalent trapezoidal fuzzy number that corresponds to the ELICIT expression $x_{u-i n d e x(i)}$ and $\zeta$ is the inverse function of $\zeta^{-1}$.

Similar to the crisp weight that was computed by the linguistic quantifiers $Q$ reviewed in Section 2.2, the fuzzy weights are expressed by a fuzzy number to indicate higher uncertainty. Therefore, the fuzzy weights are computed by type-2 linguistic quantifiers $\tilde{Q}$ [32] that are based on the type-2 fuzzy sets [38]. Type-2 linguistic quantifier $\widetilde{Q}$ guided the type-1 OWA operator by the fuzzy weight $\hat{w}_{i}=\tilde{Q}_{\frac{i}{n}}-\tilde{Q}_{\frac{i-1}{n}}, i=1, \cdots, n$. Especially, if the secondary membership function is equal to 1 , the linguistic type-2 quantifier is called the interval-valued type-2 linguistic quantifier, and it then computed the interval weight as

$$
\widetilde{w}_{i}=\left(J_{\frac{i}{n}}-J_{\frac{i-1}{n}}\right) \bigcap[0,1]
$$

where $J_{r}$ is the primary membership of the variable $r, \forall r \in[0,1]$ for all $i=1, \cdots, n$.

Figure 2 depicts the footprint of uncertainty (FOU) of the interval-valued type-2 linguistic quantifier "most".

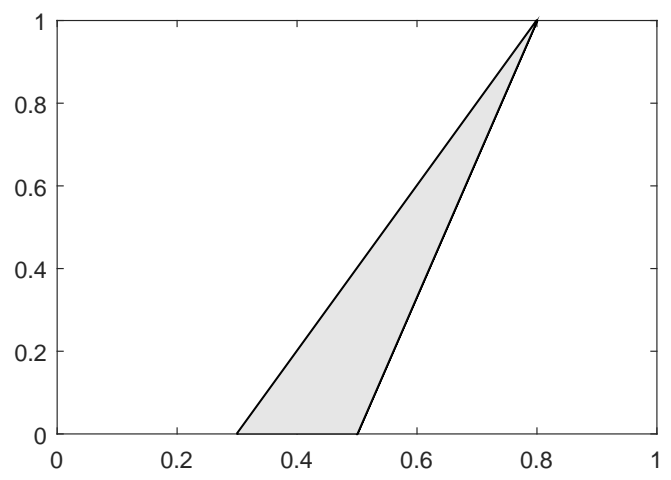

Figure 2. The FOU of the interval-valued type-2 linguistic quantifier "most".

As aforementioned, the fusion of the ELICIT information is equivalent to the fusion of the trapezoidal fuzzy number and it considers the general mathematical multiplication and division of trapezoidal fuzzy numbers that are not trapezoid preserving [34], which is not conducive to completing the ELICIT-CW approach. Therefore, it needs an approximation process to obtain the trapezoidal fuzzy number before the last re-translation process. Inspired by the type-1 OWA operator [32,37], we shall apply the EKM algorithm $[39,40]$ 
upon the $\alpha$-cut to implement the ELICIT-t1-IOWA operator with interval weight $\widetilde{w}_{i}$. After that, we will obtain an approximated result of $\sum_{i=1}^{n} \frac{\widetilde{w}_{i} \otimes \zeta^{-1}\left(x_{u-i n d e x}(i)\right.}{\sum_{i=1}^{n} \widetilde{w}_{i}}$.

For sake of clarity, let $A_{i}=\zeta^{-1}\left(x_{u-i n d e x(i)}\right)=T\left(a_{i}, b_{i}, c_{i}, d_{i}\right)$ and interval weight $\widetilde{w}_{i}$ is computed by the interval-valued type- 2 linguistic quantifier "most", we shall compute $\sum_{i=1}^{n} \frac{\widetilde{w}_{i} \otimes A_{i}}{\sum_{i=1}^{n} \widetilde{w}_{i}}$ utilizing idea of the EKM algorithm $[39,40]$ and $\alpha$ - level set theory.

In order to do this, simplify the process as the following one.

\section{- $\quad$ Step 1: Initialization}

(1) Given the interval weights $\left\{\widetilde{w}_{i}\right\}_{i=1}^{n}$ with $\widetilde{w}_{i}=\left[\omega_{i}^{L}, \omega_{i}^{R}\right]$ for $\omega_{i}^{L}, \omega_{i}^{R}$ are two endpoints of the interval weight $\widetilde{w}_{i}$ and ordered fuzzy arguments $\left\{A_{i}\right\}_{i=1}^{n}$ that are defined on the domain $[0,1]$ for all $i=1, \cdots, n$.

(2) For simplified the process, let $A_{i}$ be a trapezoidal fuzzy number as $A_{i}=$ $T\left(a_{i}, b_{i}, c_{i}, d_{i}\right)$, and then the $\alpha$ - cut of them as:

$$
\widetilde{w}_{i}^{\alpha}=\widetilde{w}_{i}=\left\{\omega \in[0,1] \mid \mu_{\widetilde{w}_{i}}(\omega) \geq \alpha\right\}=\left[\omega_{i}^{L}, \omega_{i}^{R}\right]
$$

and

$$
A_{i}^{\alpha}=\left\{y \in[0,1] \mid \mu_{A_{i}}(y) \geq \alpha\right\}=\left[a_{i}+\alpha\left(b_{i}-a_{i}\right), d_{i}-\alpha\left(d_{i}-c_{i}\right)\right]
$$

(3) Let $\boldsymbol{\Phi}^{\alpha}\left(A_{1}^{\alpha}, \cdots, A_{n}^{\alpha}\right)=A^{\alpha}$ :

$$
\boldsymbol{\Phi}^{\alpha}\left(A_{1}^{\alpha}, \cdots, A_{n}^{\alpha}\right)=A^{\alpha}=\left\{\frac{\sum_{i=1}^{n} \omega_{i} y_{i}}{\sum_{i=1}^{n} \omega_{i}} \mid \forall \omega_{i} \in \widetilde{w}_{i}, y_{i} \in A_{i}^{\alpha}\right\}=\left[L_{y^{\alpha}}, R_{y^{\alpha}}\right]
$$

where $L_{y^{\alpha}}=\min _{\forall \omega_{i} \in \widetilde{\mathfrak{w}}_{i}, y_{i} \in A_{i}^{\alpha}} \frac{\sum_{i=1}^{n} \omega_{i} y_{i}}{\sum_{i=1}^{n} \omega_{i}}$ and $R_{y^{\alpha}}=\max _{\forall \omega_{i} \in \widetilde{\mathfrak{w}}_{i}, y_{i} \in A_{i}^{\alpha}} \frac{\sum_{i=1}^{n} \omega_{i} y_{i}}{\sum_{i=1}^{n} \omega_{i}}$ are two endpoints of $A^{\alpha}$.

Additionally, $L_{y^{\alpha}}, R_{y^{\alpha}}$ represent the lower and upper endpoints of interval $A^{\alpha}$, respectively.

(4) Let $\left\langle a_{i}+\alpha\left(b_{i}-a_{i}\right), \omega_{i}\right\rangle$ be a two tuple and let $\tau:\{1, \cdots, n\} \longrightarrow\{1, \cdots, n\}$ be a permutation that only acts on the first item of the two tuple, such that $a_{\tau(i+1)}+$ $\alpha\left(b_{\tau(i+1)}-a_{\tau(i+1)}\right) \leq a_{\tau(i)}+\alpha\left(b_{\tau(i)}-a_{\tau(i)}\right)$, then $\left\langle a_{\tau(i)}+\alpha\left(b_{\tau(i)}-a_{\tau(i)}\right), \omega_{\tau(i)}\right\rangle$ be the reordered two tuple with $a_{\tau(i)}+\alpha\left(b_{\tau(i)}-a_{\tau(i+1)}\right)$ is the $i^{\text {th }}$ smallest elements in the set $\left\{a_{i}+\alpha\left(b_{i}-a_{i}\right)\right\}_{i=1}^{n}$. It is the same to deal with $\left\langle d_{i}-\alpha\left(d_{i}-c_{i}\right), \omega_{i}\right\rangle$. It helps to more easily implement the EKM algorithm.

- Step 2: To obtain the initial lower bounded $L_{y^{\alpha}}$ of $A^{\alpha}$

(1) Set $K=\left[\frac{n}{2.4}\right][39,40]$ (the nearest integer to $\frac{n}{2.4}$ ) and compute:

$$
\begin{gathered}
l_{1}=\Sigma_{i=1}^{K} \omega_{\tau(i)}^{R}\left[a_{\tau(i)}+(1-\alpha) b_{\tau(i)}\right]+\sum_{i=K+1}^{n} \omega_{\tau(i)}^{L}\left[a_{\tau(i)}+(1-\alpha) b_{\tau(i)}\right] \\
l_{2}=\Sigma_{i=1}^{K} \omega_{\tau(i)}^{R}+\sum_{i=K+1}^{n} \omega_{\tau(i)}^{L} \\
y^{\alpha}(l)=\frac{l_{1}}{l_{2}}
\end{gathered}
$$

(2) Find $K^{L} \in\{1,2, \cdots, n-1\}$, such that

$$
\left[a_{\tau\left(K^{L}\right)}+(1-\alpha) b_{\tau\left(K^{L}\right)}\right] \leq y^{\alpha}(l) \leq\left[a_{\tau\left(K^{L}+1\right)}+(1-\alpha) b_{\tau\left(K^{L}+1\right)}\right]
$$


(3) Check if $K^{L}=K$. If yes, stop and set $y^{\alpha}(l)=L_{y^{\alpha}}$. If no, go to Step 2 (4);

(4) compute $\mathcal{S}=\operatorname{sign}\left(K^{L}-K\right)=\left\{\begin{array}{l}1, \text { if } K^{L}-K>0 \\ 0, \text { if } K^{L}-K=0, \text { and } \\ -1, \text { if } K^{L}-K<0\end{array}\right.$

$$
\begin{gathered}
l_{11}=l_{1}+\mathcal{S} \Sigma_{i=\min \left(K, K^{L}\right)}^{\max \left(K, K^{L}\right)}\left[a_{\tau(i)}+(1-\alpha) b_{\tau(i)}\right]\left(\omega_{\tau(i)}^{R}-\omega_{\tau(i)}^{L}\right) \\
l_{22}=l_{2}+\mathcal{S} \Sigma_{i=\min \left(K, K^{L}\right)}^{\max \left(K, K^{L}\right)}\left(\omega_{\tau(i)}^{R}-\omega_{\tau(i)}^{L}\right) \\
y^{\alpha}(l)=\frac{l_{11}}{l_{22}}
\end{gathered}
$$

and go to Step 2(2);

- Step 3: To obtain the initial upper bounded $R_{y^{\alpha}}$ of $A^{\alpha}$

(1) Set $K=\left[\frac{n}{1.7}\right][39,40]$ (the nearest integer to $\frac{n}{1.7}$ ) and compute:

$$
\begin{gathered}
r_{1}=\sum_{i=1}^{K} \omega_{\tau(i)}^{L}\left[d_{\tau(i)}-\alpha\left(d_{\tau(i)}-c_{\tau(i)}\right)\right]+\sum_{i=K+1}^{n} \omega_{\tau(i)}^{R}\left[d_{\tau(i)}-\alpha\left(d_{\tau(i)}-c_{\tau(i)}\right)\right] \\
r_{2}=\sum_{i=1}^{K} \omega_{\tau(i)}^{L}+\sum_{i=K+1}^{n} \omega_{\tau(i)}^{R} \\
y^{\alpha}(r)=\frac{r_{1}}{r_{2}}
\end{gathered}
$$

(2) Find $K^{R} \in\{1,2, \cdots, n-1\}$, such that

$$
\left[d_{\tau\left(K^{R}\right)}-\alpha\left(d_{\tau\left(K^{R}\right)}-c_{\tau\left(K^{R}\right)}\right)\right] \leq y^{\alpha}(r) \leq\left[d_{\tau\left(K^{R}+1\right)}-\alpha\left(d_{\tau\left(K^{R}+1\right)}-c_{\tau\left(K^{R}+1\right)}\right)\right]
$$

(3) Check if $K^{R}=K$. If yes, stop and set $y^{\alpha}(r)=R_{y^{\alpha}}$. If no, go to Step 3 (4);

(4) compute $\mathcal{S}=\operatorname{sign}\left(K^{R}-K\right)=\left\{\begin{array}{l}1, \text { if } K^{R}-K>0 \\ 0, \text { if } K^{R}-K=0, \text { and } \\ -1, \text { if } K^{R}-K<0\end{array}\right.$.

$$
\begin{gathered}
r_{11}=r_{1}-\mathcal{S} \Sigma_{i=\min \left(K, K^{R}\right)}^{\max \left(K, K^{R}\right)}\left[d_{\tau(i)}-\alpha\left(d_{\tau(i)}-c_{\tau(i)}\right)\right]\left(\omega_{\tau(i)}^{R}-\omega_{\tau(i)}^{L}\right) \\
r_{22}=r_{2}-\mathcal{S} \Sigma_{i=\min \left(K, K^{R}\right)}^{\max \left(K, K^{R}\right)}\left(\omega_{\tau(i)}^{R}-\omega_{\tau(i)}^{L}\right) \\
y^{\alpha}(r)=\frac{r_{11}}{r_{22}}
\end{gathered}
$$

and go to Step 3(2); 
- $\quad$ Step 4: To obtain the final result $A^{\alpha}, A$ and $\mu_{A}(y)$;

$$
\begin{aligned}
A^{\alpha} & =\left[L_{y^{\alpha}}, R_{y^{\alpha}}\right] \\
A & =\bigcup_{0 \leq \alpha \leq 1} \alpha A^{\alpha} \\
\mu_{A}(y) & =\bigvee_{\alpha: y \in A^{\alpha}} \alpha
\end{aligned}
$$

where $L_{y^{\alpha}}=\min _{\forall \omega_{i} \in \widetilde{w}_{i}, y_{i} \in A_{i}^{\alpha}} \frac{\sum_{i=1}^{n} \omega_{i} y_{i}}{\sum_{i=1}^{n} \omega_{i}}$ and $R_{y^{\alpha}}=\max _{\forall \omega_{i} \in \widetilde{w}_{i}, y_{i} \in A_{i}^{\alpha}} \frac{\sum_{i=1}^{n} \omega_{i} y_{i}}{\sum_{i=1}^{n} \omega_{i}}$.

- $\quad$ Step 5: To execute an approximation of $A$ as a trapezoidal fuzzy number $T(a, b, c, d)$ According to literature [34,41] regarding the operation of trapezoidal fuzzy numbers, we know that the result of $A$ is a generalized trapezoidal fuzzy number, so we need to approximate it with trapezoidal fuzzy number $T(a, b, c, d)$.

Let $\alpha=0$, then we will obtain $A_{i}^{\alpha}=\left[a_{i}, d_{i}\right]$ and

$$
\begin{aligned}
& a^{\prime}=L_{y^{0}}=\min _{\forall \omega_{i} \in \widetilde{w}_{i}} \frac{\sum_{i=1}^{n} \omega_{i} a_{i}}{\sum_{i=1}^{n} \omega_{i}} \\
& d^{\prime}=R_{y^{0}}=\max _{\forall \omega_{i} \in \widetilde{w}_{i}} \frac{\sum_{i=1}^{n} \omega_{i} d_{i}}{\sum_{i=1}^{n} \omega_{i}}
\end{aligned}
$$

Let $\alpha=1$, and then we can obtain $A_{i}^{\alpha}=\left[b_{i}, c_{i}\right]$ and

$$
\begin{aligned}
b^{\prime} & =L_{y^{1}}=\min _{\forall \omega_{i} \in \widetilde{w}_{i}} \frac{\sum_{i=1}^{n} \omega_{i} b_{i}}{\sum_{i=1}^{n} \omega_{i}} \\
c^{\prime}=R_{y^{1}} & =\max _{\forall \omega_{i} \in \widetilde{w}_{i}} \frac{\sum_{i=1}^{n} \omega_{j} c_{i}}{\sum_{i=1}^{n} \omega_{i}}
\end{aligned}
$$

When considering that the area of the trapezoidal membership function graph can represent the size of the information carried, then $\exists \epsilon \in\left(-\frac{1}{2 g}, \frac{1}{2 g}\right)$, where $g+1$ is the granularity of the linguistic term set $S$ used for the ELICIT expression, such that

$$
\left|\int_{0}^{1}\left[L_{y^{\alpha}}, R_{y^{\alpha}}\right] d \alpha-\frac{1}{2}(a+b+c+d)\right| \rightarrow 0
$$

with

$$
\left\{\begin{array}{c}
\left|a-a^{\prime}\right|<\epsilon \\
b=b^{\prime} \\
c=c^{\prime} \\
\left|d-d^{\prime}\right|<\epsilon
\end{array}\right.
$$

Let $\varphi(\alpha)=\int_{0}^{1}\left[L_{y^{\alpha}}, R_{y^{\alpha}}\right] d \alpha-\frac{1}{2}(a+b+c+d)$, since $\int_{0}^{1}[a+\alpha(b-a), d-\alpha(d-c)] d \alpha$ $=\frac{1}{2}(a+b+c+d)$, then

$$
\begin{aligned}
\varphi(\alpha) & =\int_{0}^{1}\left\{L_{y^{\alpha}}-[a+\alpha(b-a)]\right\} d \alpha+\int_{0}^{1}\left\{R_{y^{\alpha}}-[d-\alpha(d-c)]\right\} d \alpha \\
& =\int_{0}^{1}\left\{\min _{\forall \omega_{i} \in \widetilde{w}_{i}} \frac{\sum_{i=1}^{n} \omega_{i}\left[a_{i}+\alpha\left(b_{i}-a_{i}\right)\right]}{\sum_{i=1}^{n} \omega_{i}}-[a+\alpha(b-a)]\right\} d \alpha \\
& +\int_{0}^{1}\left\{\max _{\forall \omega_{i} \in \widetilde{w}_{i}} \frac{\sum_{i=1}^{n} \omega_{i}\left[d_{i}-\alpha\left(d_{i}-c_{i}\right)\right]}{\sum_{i=1}^{n} \omega_{i}}-[d-\alpha(d-c)]\right\} d \alpha \\
& =\frac{1}{2}\left\{\min _{\forall \omega_{i} \in \widetilde{w}_{i}} \frac{\sum_{i=1}^{n} \omega_{i}\left(a_{i}+b_{i}\right)}{\sum_{i=1}^{n} \omega_{i}}-(b+a)\right\}+\frac{1}{2}\left\{\max _{\forall \omega_{i} \in \widetilde{w}_{i}} \frac{\sum_{i=1}^{n} \omega_{i}\left(d_{i}+c_{i}\right)}{\sum_{i=1}^{n} \omega_{i}}-(d+c)\right\}
\end{aligned}
$$


Because $\widetilde{w}_{i}$ and $A_{i}$ are defined on $[0,1]$, then

$$
\begin{aligned}
\min _{\forall \omega_{i} \in \widetilde{\mathfrak{w}}_{i}} \frac{\sum_{i=1}^{n} \omega_{i}\left(a_{i}+b_{i}\right)}{\sum_{i=1}^{n} \omega_{i}} & =\min _{\forall \omega_{i} \in \widetilde{w}_{i}} \frac{\sum_{i=1}^{n} \omega_{i} a_{i}}{\sum_{i=1}^{n} \omega_{i}}+\min _{\forall \omega_{i} \in \widetilde{\mathfrak{w}}_{i}} \frac{\sum_{i=1}^{n} \omega_{i} b_{i}}{\sum_{i=1}^{n} \omega_{i}} \\
& =a^{\prime}+b^{\prime}
\end{aligned}
$$

and

$$
\begin{aligned}
\max _{\forall \omega_{i} \in \widetilde{\mathfrak{w}}_{i}} \frac{\sum_{i=1}^{n} \omega_{i}\left(d_{i}+c_{i}\right)}{\sum_{i=1}^{n} \omega_{i}} & =\max _{\forall \omega_{i} \in \widetilde{w}_{i}} \frac{\sum_{i=1}^{n} \omega_{i} d_{i}}{\sum_{i=1}^{n} \omega_{i}}+\max _{\forall \omega_{i} \in \widetilde{w}_{i}} \frac{\sum_{i=1}^{n} \omega_{i} c_{i}}{\sum_{i=1}^{n} \omega_{i}} \\
& =c^{\prime}+d^{\prime}
\end{aligned}
$$

So far, we obtain that

$$
\varphi(\alpha)=\frac{1}{2}\left(a^{\prime}-a+d^{\prime}-d\right)
$$

Therefore, $\exists \epsilon=0 \in\left(-\frac{1}{2 g}, \frac{1}{2 g}\right)$, such that

$$
a^{\prime}=a, d^{\prime}=d \text { and }|\varphi(\alpha)|=0
$$

In general, the approximation of $A$ is the trapezoidal function $T(a, b, c, d)=T\left(a^{\prime}, b^{\prime}, c^{\prime}, d^{\prime}\right)$.

\subsection{The Order Inducing Variable in the Form of Elicit Expression}

Yager and Filev [27] introduced the idea of obtaining the order inducing variable, which is, $\Omega$ is any target set containing linear order, which can be shown in detail as follows:

More generally, we see, that if $\Omega$ is any set of objectives such that there exists a linear ordering on $\Omega$, for any distinct $x, y \in \Omega$, then either $x<y$ or $x>y$, but not both, then we can draw our the order inducing variable $u_{i}$ value from $\Omega$.

As the name stipulates, the order inducing variables are used to reorder the argument variables, when considering that the arguments have no inherent order. There are various types of the order inducing variable, for instance, $\Omega=\mathbb{R}$ for $\mathbb{R}$ is the set of all real numbers and it has an incontrovertible ordering, $\Omega$ is the implicit lexicographic order and, even while using the linguistic term set $S=\left\{S_{0}, S_{1}, \cdots, S_{g}\right\}$, where $S_{i}>S_{j}$ is for $i>j$, etc.. However, $\Omega=\mathcal{F}$ and $\Omega=\Psi$ are not often chosen, where $\mathcal{F}$ is the set of all possible ELICIT expressions, and $\Psi$ is the set of all fuzzy numbers. The reason is that, when compared with $\mathbb{R}$, there is no standard ranking method for fuzzy numbers to obtain undisputed ordering results and, considering that, on the basis of the function $\zeta^{-1}, \mathcal{F}$ can be transformed into a subset of $\Psi$, i.e., $\zeta^{-1}(\mathcal{F}) \subseteq \Psi$. Therefore, we prefer to choose $\Omega$, which contains an inherent order.

There is much literature on fuzzy number ranking, but different fuzzy number ranking methods will provide different ranking results. Based on the work of Wang and Kerre [42,43], the ranking of fuzzy numbers can be roughly divided into the following three categories:

(i) the defuzzification-based method, for instance, centroid index (CI) method [26,44], the area method, so-called the magnitude function [45], and so on;

(ii) method that measuring of the distance to the reference set [42,46,47]; and,

(iii) pairwise comparison method [43,48,49].

The purpose of all these methods is to obtain a numerical scale for ranking fuzzy numbers. Because there are many fuzzy numbers, such as interval fuzzy numbers, triangular fuzzy numbers, trapezoidal fuzzy numbers, Gaussian fuzzy numbers, and L-R type, etc., the order inducing variables only need to select one of the fuzzy numbers and one ranking method, and then the reordering of the argument variables is determined. 
Inspired by the concept of numerical scale function NS in [50], in which the function $N S: S \longrightarrow \mathbb{R}$ defines a numerical scale on the linguistic term set $S=\left\{S_{0}, S_{1}, \cdots, S_{g}\right\}$. When assuming that the OWA pair belongs to the Cartesian Product set of dimension $n,(\mathcal{F} \times \mathcal{F})^{n}$, is denoted as $\left(\left\langle u_{i}, x_{i}\right\rangle\right)_{i=1}^{n}$, then the $u_{i}$ can be obtained by using $\Delta^{-1}\left(\left(S_{i}, \alpha\right)^{\gamma}\right) \triangleq \Delta^{-1}\left(\left(S_{i}, \alpha\right)\right)=$ $i+\alpha$ [16] to define the vertex of the triangle fuzzy numbers $\left(S_{i}, \alpha\right)^{\gamma}$ without considering the adjustment parameter $\gamma$ [15], where $g+1$ is the granularity of the linguistic terms set $\left\{S_{0}, S_{1}, \cdots, S_{g}\right\}$ for all $i=0, \cdots, \mathrm{g}$, as proposed in [16]. The definition is the following one:

Definition 11. Let $\mathcal{F}$ be the set of all possible ELICIT expressions, then there is a mapping $\phi: \mathcal{F} \longrightarrow \mathbb{R}$ that is defined by the middle position of vertexes of ELICIT expressions, such that

$$
\phi\left(u_{i}\right)=\left\{\begin{array}{l}
\frac{i+\alpha+g}{2}, \text { if at least }\left(S_{i}, \alpha\right)^{\gamma} \\
\frac{i+\alpha}{2}, \text { if at most }\left(S_{i}, \alpha\right)^{\gamma} \\
\frac{i+\alpha_{1}+j+\alpha_{2}}{2}, \text { if between }\left(S_{i}, \alpha_{1}\right)^{\gamma_{1}} \text { and }\left(S_{j}, \alpha_{2}\right)^{\gamma_{2}} \\
i+\alpha, \text { if }\left(S_{i}, \alpha\right)^{\gamma}
\end{array}\right.
$$

where $S=\left\{s_{0}, s_{1}, \cdots, s_{g}\right\}$ is a set of linguistic terms and $g+1$ is the granular of the set $S$.

More generally, a mapping $\phi: \mathcal{F} \longrightarrow[0,1]$ can be defined as an extension of the numerical scalar function NS as the following formula:

$$
\phi\left(u_{i}\right)=\left\{\begin{array}{l}
0, \quad \text { if } u_{i}=S_{0} ; \\
\in\left[\frac{i-1+\alpha}{g}, 1\right], \quad \text { if } u_{i}=\text { at least }\left(S_{i}, \alpha\right)^{\gamma}, i=1, \cdots, g ; \\
\in\left[0, \frac{i+1+\alpha}{g}\right], \quad \text { if } u_{i}=\text { at most }\left(S_{i}, \alpha\right)^{\gamma}, i=0, \cdots, g-1 ; \\
\frac{1}{2}, \quad \text { if } u_{i}=S_{g} ; \\
\in\left[\frac{i-1+\alpha_{1}}{g}, \frac{j+1+\alpha_{2}}{g}\right], \quad \text { if } u_{i}=\text { between }\left(S_{i}, \alpha_{1}\right)^{\gamma_{1}} \text { and }\left(S_{j}, \alpha_{2}\right)^{\gamma_{2}}, i<j, i=1, \cdots, g, j=0, \cdots, g-1 ; \\
\in\left[\frac{i-1+\alpha}{g}, \frac{i+1+\alpha}{g}\right], \quad \text { if } u_{i}=\left(S_{i}, \alpha\right)^{\gamma}, i=1, \cdots, g-1 \\
1, \quad \text { if } u_{i}=S_{g} ;
\end{array}\right.
$$

Example 3. Assume that OWA pairs $\left(\left\langle u_{i}, x_{i}\right\rangle\right)_{i=1}^{n}$ under the linguistic terms set $\left\{S_{0}, S_{1}, S_{2}, S_{3}\right.$, $\left.S_{4}, S_{5}, S_{6}\right\}$ are noted as $\left(\left\langle\right.\right.$ at least $\left.S_{4}, x_{1}\right\rangle,\left\langle\right.$ at most $\left.S_{2}, x_{2}\right\rangle,\left\langle S_{5}, x_{3}\right\rangle,\left\langle\right.$ between $S_{4}$ and $\left.\left.S_{5}, x_{4}\right\rangle\right)$, then

$$
\phi\left(u_{1}\right)=5 ; \phi\left(u_{2}\right)=1 ; \phi\left(u_{3}\right)=2.5 ; \phi\left(u_{1}\right)=4.5 ;
$$

Thence, the decreasing sequence of the order inducing variable $u_{i}$ is $u_{\sigma(1)}=u_{1}, u_{\sigma(2)}=u_{4}, u_{\sigma(3)}=$ $u_{3}, u_{\sigma(4)}=u_{2}$, so that the reordered argument is $\left(x_{1}, x_{4}, x_{3}, x_{2}\right)$.

\section{The General Properties of the Elicit-Iowa Operator}

In this section, we will prove the general properties of the ELICIT-IOWA and ELICITt1-IOWA operators. First, we will review some of the properties of the IOWA operator [27], such as idempotency, commutativity, monotonicity, and boundedness, as shown below:

- Idempotency: if $a_{i}=a, \forall i=1, \cdots, n$, then

$$
\operatorname{IOWA}\left(\left\langle u_{1}, a_{1}\right\rangle, \cdots,\left\langle u_{n}, a_{n}\right\rangle\right)=\operatorname{IOWA}\left(\left\langle u_{1}, a\right\rangle, \cdots,\left\langle u_{n}, a\right\rangle\right)=a ;
$$

- Commutativity: if $\left(\left\langle u_{1}^{\prime}, a_{1}^{\prime}\right\rangle, \cdots,\left\langle u_{n}^{\prime}, a_{n}^{\prime}\right\rangle\right)$ is a permutation of $\left(\left\langle u_{1}, a_{1}\right\rangle, \cdots,\left\langle u_{n}, a_{n}\right\rangle\right)$, then

$$
\operatorname{IOWA}\left(\left\langle u_{1}, a_{1}\right\rangle, \cdots,\left\langle u_{n}, a_{n}\right\rangle\right)=\operatorname{IOWA}\left(\left\langle u_{1}^{\prime}, a_{1}^{\prime}\right\rangle, \cdots,\left\langle u_{n}^{\prime}, a_{n}^{\prime}\right\rangle\right) ;
$$


- Monotonicity: if $a_{i} \geq b_{i}$ for two OWA pairs $\left(\left\langle u_{1}, a_{1}\right\rangle, \ldots,\left\langle u_{n}, a_{n}\right\rangle\right)$ and $\left(\left\langle u_{1}, b_{1}\right\rangle, \cdots\right.$, $\left.\left\langle u_{n}, b_{n}\right\rangle\right)$, then

$$
\operatorname{IOWA}\left(\left\langle u_{1}, a_{1}\right\rangle, \cdots,\left\langle u_{n}, a_{n}\right\rangle\right) \geq \operatorname{IOWA}\left(\left\langle u_{1}, b_{1}\right\rangle, \cdots,\left\langle u_{n}, b_{n}\right\rangle\right)
$$

The condition for the establishment of the monotonicity property is that the order inducing variable $u_{i}$ is unchanged, if not this property does not necessarily hold [27].

- Boundedness: $\min _{i}\left\{a_{i}\right\} \leq \operatorname{IOWA}\left(\left\langle u_{1}, a_{1}\right\rangle, \cdots,\left\langle u_{n}, a_{n}\right\rangle\right) \leq \max _{i}\left\{a_{i}\right\}$;

It is worth noting that, because the ELICIT-IOWA operator only replaces the ELICIT expression for the real argument variable in the OWA pair of the classic IOWA operator, then the ELICIT-IOWA operator is also idempotency, commutativity, monotonicity, and boundedness. The following theorems are the property theorems of the ELICIT-IOWA operator:

Theorem 1. (Idempotency) Let $\mathbf{F}$ be an ELICIT-IOWA operator, if $x_{i}=x, i=1,2, \cdots, n$, then

$$
\mathbf{F}\left(\left\langle u_{1}, x_{1}\right\rangle, \cdots,\left\langle u_{n}, x_{n}\right\rangle\right)=x .
$$

Proof. Because $x_{i}=x$, i.e., $\zeta^{-1}\left(x_{u-\operatorname{index}(i)}\right)=\zeta^{-1}(x)=T(a, b, c, d)$ and $\sum_{i=1}^{n} \omega_{i}=1$ for all $i=1,2, \cdots, n$, then

$$
\mathbf{F}\left(\left\langle u_{1}, x_{1}\right\rangle, \cdots,\left\langle u_{n}, x_{n}\right\rangle\right)=\zeta\left(\sum_{i=1}^{n} \omega_{i} \times \zeta^{-1}(x)\right)=\zeta\left(\zeta^{-1}(x)\right)=x .
$$

Hence,

$$
\mathbf{F}\left(\left\langle u_{1}, x_{1}\right\rangle, \cdots,\left\langle u_{n}, x_{n}\right\rangle\right)=x
$$

Theorem 2. (Commutativity) Let $\mathbf{F}$ be an ELICIT-IOWA operator and $\left(\left\langle u_{1}^{\prime}, x_{1}^{\prime}{ }_{1}\right\rangle, \cdots,\left\langle u_{n}^{\prime}, x_{n}^{\prime}\right\rangle\right)$ is a permutation of $\left(\left\langle u_{1}, x_{1}\right\rangle, \cdots,\left\langle u_{n}, x_{n}\right\rangle\right)$, then

$$
\mathbf{F}\left(\left\langle u_{1}^{\prime}{ }_{1}, x_{1}^{\prime}\right\rangle, \cdots,\left\langle u_{n}^{\prime}, x_{n}^{\prime}\right\rangle\right)=\mathbf{F}\left(\left\langle u_{1}, x_{1}\right\rangle, \cdots,\left\langle u_{n}, x_{n}\right\rangle\right) .
$$

Proof. Because $\left(\left\langle u_{1}^{\prime}{ }_{1}, x_{1}^{\prime}\right\rangle, \cdots,\left\langle u_{n}^{\prime}, x_{n}^{\prime}\right\rangle\right)$ is a permutation of $\left(\left\langle u_{1}, x_{1}\right\rangle, \cdots,\left\langle u_{n}, x_{n}\right\rangle\right)$ so that for the same order inducing variable, we obtain the same reordering of the argument variable; in other words, $x_{u-i n d e x(\mathrm{i})}=x^{\prime}{ }_{u^{\prime}-i n d e x(\mathrm{i})}$ for all $i=1, \cdots, n$, then

$$
\begin{aligned}
\mathbf{F}\left(\left\langle u_{1}, x_{1}\right\rangle, \cdots,\left\langle u_{n}, x_{n}\right\rangle\right) & =\zeta\left(\sum_{i=1}^{n} \omega_{i} \times \zeta^{-1}\left(x_{u-i n d e x(\mathrm{i})}\right)\right) \\
& =\zeta\left(\sum_{i=1}^{n} \omega_{i} \times \zeta^{-1}\left({x^{\prime}}_{u^{\prime}-\operatorname{index}(\mathrm{i})}\right)\right) \\
& =F\left(\left\langle u^{\prime}, x^{\prime}{ }_{1}\right\rangle, \cdots,\left\langle u^{\prime}{ }_{n}, x^{\prime}{ }_{n}\right\rangle\right) .
\end{aligned}
$$

Therefore,

$$
\mathbf{F}\left(\left\langle u_{1}^{\prime}{ }_{1}, x_{1}^{\prime}\right\rangle, \cdots,\left\langle u_{n}^{\prime}, x_{n}^{\prime}\right\rangle\right)=\mathbf{F}\left(\left\langle u_{1}, x_{1}\right\rangle, \cdots,\left\langle u_{n}, x_{n}\right\rangle\right) .
$$

As we all know, the set $\mathcal{F}$ (be a set of all possible ELICIT expressions) has no inherent order due to its fuzzy representation. Therefore, we can choose a predefined numeric scalar function $\phi: \mathcal{F} \longrightarrow \mathbb{R}$ (Section 3.3) to order the elements. In this order, the distinct variables $x_{i}, x_{j} \in \mathcal{F}$ 
are comparable, which is, only $x_{i} \prec x_{j}$ or $x_{i} \succ x_{j}$, but neither simultaneously exist. At the same time, we will obtain that $\omega \times \zeta^{-1}\left(x_{i}\right)>\omega \times \zeta^{-1}\left(x_{j}\right)$ or $\omega \times \zeta^{-1}\left(x_{i}\right)<\omega \times \zeta^{-1}\left(x_{j}\right), \forall \omega \in[0,1]$, but not both.

Theorem 3. (Monotonicity) Let $\mathbf{F}$ be an ELICIT-IOWA operator. $\left(\left\langle u_{1}, x_{1}\right\rangle, \cdots,\left\langle u_{n}, x_{n}\right\rangle\right)$ and $\left(\left\langle u_{1}, z_{1}\right\rangle, \cdots,\left\langle u_{n}, z_{n}\right\rangle\right)$ are two sequences of ELICIT-based OWA pairs, then

$$
\mathbf{F}\left(\left\langle u_{1}, z_{1}\right\rangle, \cdots,\left\langle u_{n}, z_{n}\right\rangle\right) \preceq \mathbf{F}\left(\left\langle u_{1}, x_{1}\right\rangle, \cdots,\left\langle u_{n}, x_{n}\right\rangle\right)
$$

for all $z_{i} \prec x_{i}$ under the same order inducing variable $u_{i},(i=1, \cdots, n)$.

Proof. Because $z_{i} \prec x_{i}$ under the same order inducing variable $u_{i}$, we will obtain $z_{u-i n d e x(i)}$ $\prec x_{u-\operatorname{index}(i)}$ and $\zeta^{-1}\left(z_{u-\operatorname{index}(i)}\right)<\zeta^{-1}\left(x_{u-\operatorname{index}(i)}\right)$, such that

$$
\begin{aligned}
\mathbf{F}\left(\left\langle u_{1}, z_{1}\right\rangle, \cdots,\left\langle u_{n}, z_{n}\right\rangle\right) & =\zeta\left(\sum_{i=1}^{n} \omega_{i} \times \zeta^{-1}\left(z_{u-\operatorname{index}(i)}\right)\right) \\
& \preceq \zeta\left(\sum_{i=1}^{n} \omega_{i} \times \zeta^{-1}\left(x_{u-\operatorname{index}(i)}\right)\right) \\
& =F\left(\left\langle u_{1}, x_{1}\right\rangle, \cdots,\left\langle u_{n}, x_{n}\right\rangle\right) .
\end{aligned}
$$

Thence,

$$
\mathbf{F}\left(\left\langle u_{1}, z_{1}\right\rangle, \cdots,\left\langle u_{n}, z_{n}\right\rangle\right) \preceq \mathbf{F}\left(\left\langle u_{1}, x_{1}\right\rangle, \cdots,\left\langle u_{n}, x_{n}\right\rangle\right) .
$$

Theorem 4. (Boundedness) Let $\mathbf{F}$ be an ELICIT-IOWA operator, then

$$
\min _{i}\left\{x_{i}\right\} \preceq \mathbf{F}\left(\left\langle u_{1}, x_{1}\right\rangle, \cdots,\left\langle u_{n}, x_{n}\right\rangle\right) \preceq \max _{i}\left\{x_{i}\right\}
$$

Proof. Let $\max _{i}\left\{x_{i}\right\}=x^{*}=x_{u-\operatorname{index}(j)}$ and $\min _{i}\left\{x_{i}\right\}=x_{*}=x_{u-\text { index }(k)}$ with $j, k \in\{1, \cdots, n\}$, then

$$
\begin{aligned}
\mathbf{F}\left(\left\langle u_{1}, z_{1}\right\rangle, \cdots,\left\langle u_{n}, z_{n}\right\rangle\right) & =\zeta\left(\sum_{i=1}^{n} \omega_{i} \times \zeta^{-1}\left(x_{u-i n d e x(i)}\right)\right) \\
& \preceq \zeta\left(\sum_{i=1}^{n} \omega_{i} \times \zeta^{-1}\left(x^{*}\right)\right) \\
& =x^{*}
\end{aligned}
$$

That is

$$
\mathbf{F}\left(\left\langle u_{1}, x_{1}\right\rangle, \cdots,\left\langle u_{n}, x_{n}\right\rangle\right) \preceq x^{*} .
$$

Similarly, $F\left(\left\langle u_{1}, x_{1}\right\rangle, \cdots,\left\langle u_{n}, x_{n}\right\rangle\right) \succeq x_{*}$, then

$$
x_{*} \preceq \mathbf{F}\left(\left\langle u_{1}, x_{1}\right\rangle, \cdots,\left\langle u_{n}, x_{n}\right\rangle\right) \preceq x^{*} .
$$

Therefore,

$$
\min _{i}\left\{x_{i}\right\} \preceq \mathbf{F}\left(\left\langle u_{1}, x_{1}\right\rangle, \cdots,\left\langle u_{n}, x_{n}\right\rangle\right) \preceq \max _{i}\left\{x_{i}\right\}
$$

The process of proving the properties theorem of the ELICIT-t1-IOWA operator is similar to that of the ELICIT-IOWA operator, because the ELICIT-t1-IOWA operator is based on the extension of the ELICIT-IOWA operator, which means that the weights are fuzzy numbers instead of real numbers. Here, we will prove these properties with fuzzy 
weight $\hat{w}_{i}$ being computed by the type-2 linguistic quantifier [32]; these properties can be shown with the following theorems:

Theorem 5. (Idempotency) Let $\mathbf{F}$ be an ELICIT-t1-IOWA operator, if $x_{i}=x, i=1,2, \cdots, n$, then

$$
\mathbf{F}\left(\left\langle u_{1}, x_{1}\right\rangle, \cdots,\left\langle u_{n}, x_{n}\right\rangle\right)=x
$$

Proof. Because $x_{i}=x$, i.e., $\zeta^{-1}\left(x_{u-i n d e x(i)}\right)=\zeta^{-1}(x)=T(a, b, c, d)$ for all $i=1,2, \cdots, n$, then

$$
\mathbf{F}\left(\left\langle u_{1}, x_{1}\right\rangle, \cdots,\left\langle u_{n}, x_{n}\right\rangle\right)=\zeta\left(\frac{\sum_{i=1}^{n} \hat{w}_{i} \otimes \zeta^{-1}(x)}{\sum_{i=1}^{n} \hat{w}_{i}}\right)
$$

We need to demonstrate that $\frac{\sum_{i=1}^{n} h a t w_{i} \otimes \zeta^{-1}(x)}{\sum_{i=1}^{n} \hat{w}_{i}}=\frac{\zeta^{-1}(x) \otimes\left(\sum_{i=1}^{n} \hat{w}_{i}\right)}{\sum_{i=1}^{n} \hat{w}_{i}}=\zeta^{-1}(x)$.

Now, we shall obtain the $\alpha$-cut of the fuzzy weight $\hat{w}_{i}$ and $\zeta^{-1}(x)=T(a, b, c, d)$, as shown below:

$$
\begin{gathered}
\hat{w}_{i}^{\alpha}=\left\{\omega \in[0,1] \mid \mu_{\hat{w}_{i}}(\omega) \geq \alpha\right\}=\left[L_{\omega_{i}^{\alpha}}, R_{\omega_{i}^{\alpha}}\right] \\
\left(\zeta^{-1}(x)\right)^{\alpha}=\left\{y \in[0,1] \mid \mu_{\zeta^{-1}(x)}(y) \geq \alpha\right\}=[a+\alpha(b-a), d-\alpha(d-c)]
\end{gathered}
$$

Let $\boldsymbol{\Phi}^{\alpha}\left(\left(\zeta^{-1}(x)\right)^{\alpha}, \cdots,\left(\zeta^{-1}(x)\right)^{\alpha}\right)=A^{\alpha}$ and $A^{\alpha}=\left\{\frac{\sum_{i=1}^{n} \omega_{i} y_{i}}{\sum_{i=1}^{n} \omega_{i}} \mid \forall \omega_{i} \in \hat{w_{i}^{\alpha}}, \forall y_{i} \in\left(\zeta^{-1}(x)\right)^{\alpha}\right\}$ $=\left[L_{y^{\alpha}}, R_{y^{\alpha}}\right]$, where $L_{y^{\alpha}}=\min _{\forall \omega_{i} \in \hat{w}_{i}^{\alpha}, y_{i} \in A_{i}^{\alpha}} \frac{\sum_{i=1}^{n} \omega_{i} y_{i}}{\sum_{i=1}^{n} \omega_{i}}$ and $R_{y^{\alpha}}=\max _{\forall \omega_{i} \in \hat{w}_{i}^{\alpha}, y_{i} \in A_{i}^{\alpha}} \frac{\sum_{i=1}^{n} \omega_{i} y_{i}}{\sum_{i=1}^{n} \omega_{i}}$, then

$$
\begin{aligned}
L_{y^{\alpha}} & =\frac{\sum_{i=1}^{i=K_{\alpha}^{L}} R_{\omega_{i}^{\alpha}}[a+\alpha(b-a)]+\sum_{i=K_{\alpha}^{L}+1}^{n} L_{\omega_{i}^{\alpha}}[a+\alpha(b-a)]}{\sum_{i=1}^{i=K_{\alpha}^{L}} R_{\omega_{i}^{\alpha}}+\sum_{i=K_{\alpha}^{L}+1}^{n} L_{\omega_{i}^{\alpha}}} \\
& =\frac{\left[\sum_{i=1}^{i=K_{\alpha}^{L}} R_{\omega_{i}^{\alpha}}+\sum_{i=K_{\alpha}^{L}+1}^{n} L_{\omega_{i}^{\alpha}}\right][a+\alpha(b-a)]}{\sum_{i=1}^{i=K_{\alpha}^{L}} R_{\omega_{i}^{\alpha}}+\sum_{i=K_{\alpha}^{L}+1}^{n} L_{\omega_{i}^{\alpha}}} \\
& =a+\alpha(b-a)
\end{aligned}
$$

We will observe that, here, $K_{\alpha}^{L}$ is no longer meaningful as a left switch point [40,51]. Simultaneously, derive $R_{y^{\alpha}}=d-\alpha(d-c)$, hence

$$
\begin{aligned}
\frac{\sum_{i=1}^{n} \hat{w}_{i} \otimes \zeta^{-1}(x)}{\sum_{i=1}^{n} h a t w_{i}} & =\bigcup_{0 \leq \alpha \leq 1} \alpha[a+\alpha(b-a), d-\alpha(d-c)] \\
& =\bigcup_{0 \leq \alpha \leq 1} \alpha\left(\zeta^{-1}(x)\right)^{\alpha} \\
& =\zeta^{-1}(x)
\end{aligned}
$$

Because

$$
\begin{aligned}
\frac{\zeta^{-1}(x) \otimes\left(\sum_{i=1}^{n} \hat{w}_{i}\right)}{\sum_{i=1}^{n} \hat{w}_{i}} & =\bigcup_{0 \leq \alpha \leq 1} \alpha\left\{\frac{y \sum_{i=1}^{n} \omega_{i}}{\sum_{i=1}^{n} \omega_{i}} \mid \forall \omega_{i} \in \hat{w}_{i}^{\alpha}, \forall y \in\left(\zeta^{-1}(x)\right)^{\alpha}\right\} \\
& =\bigcup_{0 \leq \alpha \leq 1} \alpha\left\{y \mid \forall y \in\left(\zeta^{-1}(x)\right)^{\alpha}\right\} \\
& =\zeta^{-1}(x)
\end{aligned}
$$


So far, we have already demonstrated

$$
\frac{\sum_{i=1}^{n} \hat{w}_{i} \otimes \zeta^{-1}(x)}{\sum_{i=1}^{n} \hat{w}_{i}}=\frac{\zeta^{-1}(x) \otimes\left(\sum_{i=1}^{n} \hat{w}_{i}\right)}{\sum_{i=1}^{n} \hat{w}_{i}}=\zeta^{-1}(x)
$$

Hence,

$$
\mathbf{F}\left(\left\langle u_{1}, x_{1}\right\rangle, \cdots,\left\langle u_{n}, x_{n}\right\rangle\right)=x .
$$

Theorem 6. (Commutativity) Let $\mathbf{F}$ be an ELICIT-t1-IOWA operator and $\left(\left\langle u^{\prime}{ }_{1}, x_{1}^{\prime}{ }_{1}\right\rangle, \cdots\right.$, $\left.\left\langle u_{n}^{\prime}, x_{n}^{\prime}\right\rangle\right)$ is a permutation of $\left(\left\langle u_{1}, x_{1}\right\rangle, \cdots,\left\langle u_{n}, x_{n}\right\rangle\right)$, then

$$
\mathbf{F}\left(\left\langle u_{1}^{\prime}, x_{1}^{\prime}{ }_{1}, \cdots,\left\langle u_{n}^{\prime}, x_{n}^{\prime}\right\rangle\right)=\mathbf{F}\left(\left\langle u_{1}, x_{1}\right\rangle, \cdots,\left\langle u_{n}, x_{n}\right\rangle\right) .\right.
$$

Proof. Because $\left(\left\langle u_{1}^{\prime}{ }_{1}, x_{1}^{\prime}\right\rangle, \cdots,\left\langle u_{n}^{\prime}, x_{n}^{\prime}\right\rangle\right)$ is a permutation of $\left(\left\langle u_{1}, x_{1}\right\rangle, \cdots,\left\langle u_{n}, x_{n}\right\rangle\right)$ so that for the same order inducing variable, we obtain the same reordering of the argument variable, in other words, $x_{u-i n d e x(\mathrm{i})}=x_{u^{\prime}-\text { index (i) }}^{\prime}$ for all $i=1, \cdots, n$, then

$$
\begin{aligned}
\mathbf{F}\left(\left\langle u_{1}, x_{1}\right\rangle, \ldots,\left\langle u_{n}, x_{n}\right\rangle\right) & =\zeta\left(\frac{\sum_{i=1}^{n} \hat{w}_{i} \otimes \zeta^{-1}\left(x_{u-i n d e x(i)}\right)}{\sum_{i=1}^{n} \hat{w}_{i}}\right) \\
& =\zeta\left(\frac{\sum_{i=1}^{n} \hat{w}_{i} \otimes \zeta^{-1}\left(x^{\prime}{ }_{u^{\prime}-\operatorname{index}(i)}\right)}{\sum_{i=1}^{n} \hat{w}_{i}}\right) \\
& =\mathbf{F}\left(\left\langle u^{\prime}{ }_{1}, x^{\prime}{ }_{1}\right\rangle, \cdots,\left\langle u_{n}^{\prime}{ }_{n}, x^{\prime}{ }_{n}\right\rangle\right) .
\end{aligned}
$$

Therefore,

$$
\mathbf{F}\left(\left\langle u_{1}^{\prime}{ }_{1} x_{1}^{\prime}{ }_{1}, \cdots,\left\langle u_{n}^{\prime}, x_{n}^{\prime}\right\rangle\right)=\mathbf{F}\left(\left\langle u_{1}, x_{1}\right\rangle, \cdots,\left\langle u_{n}, x_{n}\right\rangle\right) .\right.
$$

Theorem 7. (Monotonicity) Let $\mathbf{F}$ be an ELICIT-t1-IOWA operator. $\left(\left\langle u_{1}, x_{1}\right\rangle, \cdots,\left\langle u_{n}, x_{n}\right\rangle\right)$ and $\left(\left\langle u_{1}, z_{1}\right\rangle, \cdots,\left\langle u_{n}, z_{n}\right\rangle\right)$ are two sequences of ELICIT-based OWA pairs, then

$$
\mathbf{F}\left(\left\langle u_{1}, z_{1}\right\rangle, \cdots,\left\langle u_{n}, z_{n}\right\rangle\right) \preceq \mathbf{F}\left(\left\langle u_{1}, x_{1}\right\rangle, \cdots,\left\langle u_{n}, x_{n}\right\rangle\right)
$$

for all $z_{i} \prec x_{i}$ under the same order inducing variables $u_{i},(i=1, \cdots, n)$.

Proof. Because $z_{i} \prec x_{i}$ under the same order inducing variable $u_{i}$, we obtain that $z_{u-\text { index }(i)}$ $\prec x_{u-i n d e x(i)}$ and $\zeta^{-1}\left(z_{u-i n d e x(i)}\right)<\zeta^{-1}\left(x_{u-i n d e x(i)}\right)$, such that

$$
\begin{aligned}
\mathbf{F}\left(\left\langle u_{1}, z_{1}\right\rangle, \cdots,\left\langle u_{n}, z_{n}\right\rangle\right) & =\zeta\left(\frac{\sum_{i=1}^{n} \hat{w}_{i} \otimes \zeta^{-1}\left(z_{u-\operatorname{index}(i)}\right)}{\sum_{i=1}^{n} \hat{w}_{i}}\right) \\
& \preceq \zeta\left(\frac{\sum_{i=1}^{n} \hat{w}_{i} \otimes \zeta^{-1}\left(x_{u-\operatorname{index}(i)}\right)}{\sum_{i=1}^{n} \hat{w}_{i}}\right) \\
& =\mathbf{F}\left(\left\langle u_{1}, x_{1}\right\rangle, \cdots,\left\langle u_{n}, x_{n}\right\rangle\right)
\end{aligned}
$$

Hence,

$$
\mathbf{F}\left(\left\langle u_{1}, z_{1}\right\rangle, \cdots,\left\langle u_{n}, z_{n}\right\rangle\right) \preceq \mathbf{F}\left(\left\langle u_{1}, x_{1}\right\rangle, \cdots,\left\langle u_{n}, x_{n}\right\rangle\right) .
$$


Theorem 8. (Boundedness) Let $F$ be an ELICIT-t1-IOWA operator, then

$$
\min _{i}\left\{x_{i}\right\} \preceq \mathbf{F}\left(\left\langle u_{1}, x_{1}\right\rangle, \cdots,\left\langle u_{n}, x_{n}\right\rangle\right) \preceq \max _{i}\left\{x_{i}\right\} .
$$

Proof. Let $\max _{i}\left\{x_{i}\right\}=x^{*}=x_{u-i n d e x(j)}$ and $\min _{i}\left\{x_{i}\right\}=x_{*}=x_{u-i n d e x(k)}$ with $j, k \in\{1, \cdots$, $n\}$, then

$$
\begin{aligned}
\mathbf{F}\left(\left\langle u_{1}, x_{1}\right\rangle, \cdots,\left\langle u_{n}, x_{n}\right\rangle\right) & =\zeta\left(\frac{\sum_{i=1}^{n} \hat{w}_{i} \otimes \zeta^{-1}\left(x_{u-\operatorname{index}(i)}\right)}{\sum_{i=1}^{n} \hat{w}_{i}}\right) \\
& \preceq \zeta\left(\frac{\sum_{i=1}^{n} \hat{w}_{i} \otimes \zeta^{-1}\left(x_{u-i n d e x(j)}\right)}{\sum_{i=1}^{n} \hat{w}_{i}}\right) \\
& =\zeta\left(\frac{\sum_{i=1}^{n} \hat{w}_{i} \otimes \zeta^{-1}\left(x^{*}\right)}{\sum_{i=1}^{n} \hat{w}_{i}}\right) \\
& =x^{*}
\end{aligned}
$$

That is

$$
\mathbf{F}\left(\left\langle u_{1}, x_{1}\right\rangle, \cdots,\left\langle u_{n}, x_{n}\right\rangle\right) \preceq x^{*} .
$$

Similarly, $F\left(\left\langle u_{1}, x_{1}\right\rangle, \cdots,\left\langle u_{n}, x_{n}\right\rangle\right) \succeq x_{*}$, then

$$
x_{*} \preceq \mathbf{F}\left(\left\langle u_{1}, x_{1}\right\rangle, \cdots,\left\langle u_{n}, x_{n}\right\rangle\right) \preceq x^{*} .
$$

Therefore,

$$
\min _{i}\left\{x_{i}\right\} \preceq \mathbf{F}\left(\left\langle u_{1}, x_{1}\right\rangle, \cdots,\left\langle u_{n}, x_{n}\right\rangle\right) \preceq \max _{i}\left\{x_{i}\right\}
$$

\section{A Majority-Driven Gdm Process for Elicit Information}

In this section, we will propose a majority-driven GDM process for ELICIT information together with the ELICIT-t1-IOWA and ELICIT-I-IOWA operator. "Majority opinion" [29] is intended to indicate that the majority of argument variables are aggregated, because they are similar or close to the same position. Therefore, in [29], a support function is used to measure the similarity between two arguments in order to obtain the induce similarly order. Suppose that a finite set of experts $\left\{e_{1}, e_{2}, \cdots, e_{m}\right\}$ participates in the GDM problem while considering a finite set of alternatives $\left\{a_{1}, a_{2}, \cdots, a_{n}\right\}$. For the aggregation phase of the GDM selection process [52], it is divided into two parts: (1) the ELICIT-t1-IOWA operator with majority opinion to aggregate the ELICIT preference relations $\mathcal{P}^{k}=\left(p_{i j}^{k}\right)_{n \times n}$ for each expert $e_{k} ;$ (2) considering the degree of prestige $\alpha_{k}$ that is enjoyed by the expert $e_{k}$ constitutes a triple $\left\langle u_{k}, e_{k}, \alpha_{k}\right\rangle$, in which the order inducing variable $u_{k}=\alpha_{k}$ then reduce to 2-tuple $\left\langle e_{k}, \alpha_{k}\right\rangle$, and apply the ELICIT-I-IOWA operator. The majority opinion for the ELICITt1-IOWA operator is based on Zadeh's expansion principle and support function [53] to obtain the similarity function Sim to group the most similar variables into the same category, and then, before aggregating the process, they are reordered in the induced similarity order. For the ELICIT-I-IOWA operator, the linguistic quantifier "most" corresponds to most opinion upon the degree of prestige $\alpha_{k}$ of experts $e_{k}$.

To do this, the GDM problem within ELICIT information is structured as follows:

- A finite set of experts $\mathcal{E}=\left\{e_{1}, e_{2}, \cdots, e_{m}\right\}, m \geq 2$;

- A finite set of alternatives $\mathcal{A}=\left\{a_{1}, a_{2}, \cdots, a_{n}\right\}, n \geq 2$; and,

- A preference relations matrix $\mathcal{P}^{k}=\left(p_{i j}^{k}\right)_{n \times n}$ has been constructed for the $k^{\text {th }}$ expert, where $p_{i j}^{k}$ represents the evaluation of the $k^{\text {th }}$ expert. The expert expresses the preference of $a_{i}$ over $a_{j}$ in the form of CLE or ELICIT expressions. The preference relations 
$p_{i j}^{k}$ and $p_{j i}^{k}$ based on the negation operator of ELICIT information [15] and function $\Delta, \Delta^{-1}[16]$.

- $\quad$ Step 1: for each expert $e_{k}, k=1, \cdots, m$, constructing a matrix $\mathcal{P}^{k}=\left(p_{i j}^{k}\right)_{n \times n}$ with $p_{i j}^{k}$ represented by CLEs or ELICIT information;

- Step 2: to obtain the induced similarity order of the set $\left\{p_{i 1}^{k}, p_{i 2}^{k}, \cdots, p_{i j}^{k}, \cdots, p_{i n}^{k}\right\}$, which is the $i^{\text {th }}$ row of the matrix $\mathcal{P}^{k}$. It is slightly different from the classical order inducing variable, which induces the argument variable $p_{i j}^{k}$ by the similar order [29] under the concept of the "majority opinion", so that the majority of similar arguments are aggregated.

(1) Let $\mathcal{F}$ be the set of all possible ELICIT expressions, and then $p_{i j}^{k} \in \mathcal{F}$ and $\mathcal{F}$ have no inherent order. When considering the predefined function $\phi: \mathcal{F} \longrightarrow \mathbb{R}$ that aims to find the middle position of the vertex of the ELICIT expression, then we can measure the distance between two ELICIT information $p_{i j}^{k}, p_{i k}^{k}$ in order to estimate it similarly. The measure of distance is defined as follows:

$$
D\left(p_{i j}^{k}, p_{i k}^{k}\right)=\left\{\begin{array}{l}
0, \text { if } j=k ; \\
\left|\phi\left(p_{i j}^{k}\right)-\phi\left(p_{i k}^{k}\right)\right|, \text { if } j \neq k ;
\end{array}\right.
$$

With the application of Equation (37) and Equation (39) with $i^{\text {th }}$ row of the matrix $\mathcal{P}^{k}$, we will obtain the result presented in Table 1.

(2) Let $\operatorname{Sum}\left(P_{i j}^{k}\right)=-\Sigma_{k=1}^{n} D\left(p_{i j}^{k}, p_{i k}^{k}\right)$ represent the overall distance value that $p_{i j}^{k}$ over $p_{i k}^{k}, k \neq j$. If the value we obtain is smaller, it means that the distance between $p_{i j}^{k}$ and $p_{i k}^{k}, k \neq j$ is closer, which further shows that them are very similar, which is in line with the idea of "majority opinion". Additionally, at the same time, we will obtain the induced similarity order of the aggregated argument $\left\{p_{i 1}^{k}, p_{i 2}^{k}, \cdots, p_{i j}^{k}, \cdots, p_{i n}^{k}\right\}$ following the increasing lexicographic order of the pair $\operatorname{Sim}\left(P_{i j}^{k}\right)=\left(\operatorname{Sum}\left(P_{i j}^{k}\right), \phi\left(p_{i j}^{k}\right)\right)$ for all $j=1 \cdots, n$. If $\operatorname{Sim}\left(P_{i j}^{k}\right)$ has $q$ ties, then set the average of $q$ items to replace them in order to solve this problem.

- $\quad$ Step 3: using the ELICIT-t1-IOWA operator with interval weight $\widetilde{w}_{j} \subseteq[0,1]$ computed by the type-2 linguistic quantifier "most" [32] to aggregate reordered set $\left\{p_{i 1}^{k}, p_{i 2}^{k}, \cdots, p_{i j}^{k}, \cdots\right.$, $\left.p_{i n}^{k}\right\}$, noted as vector $\mathcal{M}^{k}=\left[\begin{array}{c}a_{1}^{k} \\ a_{2}^{k} \\ \vdots \\ a_{n}^{k}\end{array}\right]$ with $a_{i}^{k}=\frac{\sum_{j=1}^{n} \widetilde{w}_{j} \otimes \zeta^{-1}\left(p_{\sigma_{S i m}}^{k}(i j)\right.}{\sum_{j=1}^{n} \widetilde{w}_{j}}$ and $a_{i}^{k}$ represents the aggregated result of the $i^{\text {th }}$ alternative $a_{i}$ for the $k^{\text {th }}$ expert. Where $\sigma_{\text {Sim }}$ : $\{1, \cdots, n\} \longrightarrow\{1, \cdots, n\}$ is the permutation function on the set $\left\{p_{i 1}^{k}, p_{i 2}^{k}, \cdots, p_{i j}^{k}, \cdots\right.$, $\left.p_{i n}^{k}\right\}$ upon the induced similarity order.

- $\quad$ Step 4: utilizing the ELICIT-I-IOWA operator to aggregate $\mathcal{M}^{k}$ with the functional generated weight [35] $\omega_{k}$ for all $k=1, \cdots, m$.

Each expert $e_{k}$ is associated with a degree of prestige $\alpha_{k}=u_{k}, u_{k}$ is the order inducing variable, constituting the 2-tuple $\left\langle e_{k}, \alpha_{k}\right\rangle=\left\langle\mathcal{M}^{k}, \alpha_{k}\right\rangle$, then apply the I-ELICITIOWA operator with linguistic quantifier "most" so that the BUM function $Q(r)$ with 
$\alpha=0.3, \beta=0.8$, we will obtain the result $\mathcal{R}=\Sigma_{k=1}^{m} \omega_{k} \times \mathcal{M}^{\sigma(k)}=\left[\begin{array}{c}\beta_{1} \\ \beta_{2} \\ \vdots \\ \beta_{n}\end{array}\right]$ with $\beta_{i}=\Sigma_{k=1}^{m} \omega_{k} \times a_{i}^{\sigma(k)}$ and $\omega_{k}=Q\left(\frac{I(k)}{I(m)}\right)-Q\left(\frac{I(k-1)}{I(m)}\right)$, where $I(k)=\Sigma_{j=1}^{k} \alpha_{\sigma(j)}, j, k=1, \cdots$, $m$ and $\sigma:\{1, \cdots, m\} \longrightarrow\{1, \cdots, m\}$ is permutation function only acting on the variable $\alpha_{k}$, such that $\alpha_{\sigma(k)}$ is the $i^{t h}$ largest element of set $\left\{\alpha_{1}, \cdots, \alpha_{m}\right\}$, then the noted corresponding reordered 2-tuple as $\left(\left\langle\mathcal{M}^{\sigma(1)}, \alpha_{\sigma(1)}\right\rangle, \cdots,\left\langle\mathcal{M}^{\sigma(m)}, \alpha_{\sigma(m)}\right\rangle\right)$.

- Step 5: ranking the element of set $\left\{\beta_{1}, \beta_{2}, \cdots, \beta_{n}\right\}$ in order to select the top one $\beta_{i}$ corresponding to the alternative $a_{i}$ as the solution of the GDM problem and obtaining the final result with function $\zeta$ as $\zeta(\mathcal{R})=\left[\begin{array}{c}\zeta\left(\beta_{1}\right) \\ \zeta\left(\beta_{2}\right) \\ \vdots \\ \zeta\left(\beta_{n}\right)\end{array}\right]$ in the form of ELICIT expressions.

Table 1. The overall distance measure for the $i^{\text {th }}$ row of the matrix $\mathcal{P}^{k}$.

\begin{tabular}{ccccccc}
\hline$i^{\text {th }}$ row & $p_{i 1}^{k}$ & $\cdots$ & $p_{i j}^{k}$ & $\cdots$ & $p_{i n}^{k}$ & Sum \\
\hline$p_{i 1}^{k}$ & 0 & & & & & \\
\hline$\vdots$ & & & & & & \\
\hline$p_{i j}^{k}$ & & 0 & & & \\
\hline$\vdots$ & & & & \\
\hline$p_{i n}^{k}$ & & & & & \\
\hline
\end{tabular}

\section{Illustrative Example}

This section introduces an illustrative example in order to show the performance of our proposal and its feasibility in the GDM problem with ELICIT information. In addition, it provides a brief comparative analysis to show the advantages of the ELICIT-IOWA operators that were introduced regarding previous ELICIT aggregation operators for dealing with GDM problems.

\subsection{Gdm Problem with Elicit Information}

Let us assume that this GDM problem involves five experts $\mathcal{E}=\left\{e_{1}, e_{2}, e_{3}, e_{4}, e_{5}\right\}$ and four alternatives $\mathcal{A}=\left\{a_{1}, a_{2}, a_{3}, a_{4}\right\}$.

Each expert provides his or her ELICIT preference relation while using ELICIT expressions that were built by using the linguistic term set $S=\left\{S_{0}, S_{1}, S_{2}, S_{3}, S_{4}, S_{5}, S_{6}, S_{7}, S_{8}\right\}$, whose semantics are $S_{0}=$ None; $S_{1}=$ Very Low; $S_{2}=$ Low; $S_{3}=$ Slightly Low; $S_{4}=$ Medium; $S_{5}=$ Slightly High; $S_{6}=$ High; $S_{7}=$ Very High; $S_{8}=$ Perfect and its semantics are shown in Figure 3.

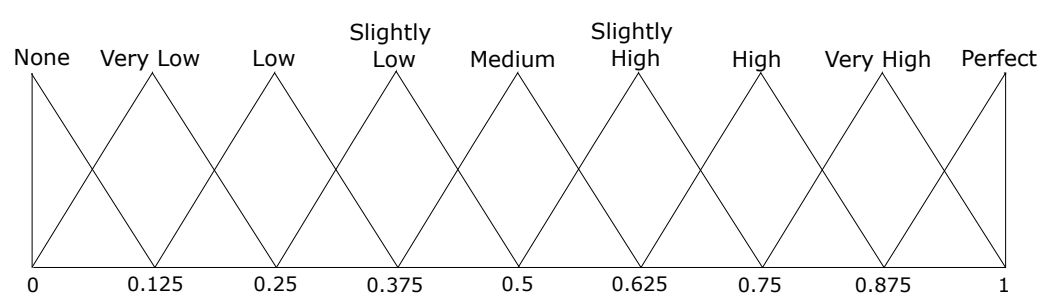

Figure 3. The ELICIT expression examples. 


\subsection{A Majority-Driven Solving Process Driven by Elicit-T1-Owa and Elicit-I-Iowa with Most} Linguistic Quantifier

The Majority-driven GDM process for ELICIT information that is presented in Section 5 is applied to solve the previous problem.

- $\quad$ Step 1: the five ELICIT preference relation matrix $\mathcal{P}^{k}$ are provided as follows:

$$
\begin{aligned}
& \mathcal{P}^{1}=\left[\begin{array}{cccc}
s_{4} & \text { at most } s_{3} & \text { between } s_{6} \text { and } s_{7} & s_{1} \\
\text { at least } s_{5} & s_{4} & \text { at least } s_{6} & \text { at least } s_{6} \\
\text { between } s_{1} \text { and } s_{2} & \text { at most } s_{2} & s_{4} & \text { between } s_{2} \text { and } s_{3} \\
s_{7} & \text { at most } s_{2} & \text { between } s_{5} \text { and } s_{6} & s_{4}
\end{array}\right] \\
& \mathcal{P}^{2}=\left[\begin{array}{cccc}
s_{4} & s_{5} & \text { between } s_{6} \text { and } s_{7} & \text { between } s_{2} \text { and } s_{3} \\
s_{3} & s_{4} & \text { at most } s_{1} & \text { at least } s_{7} \\
\text { between } s_{1} \text { and } s_{2} & \text { at least } s_{7} & s_{4} & \text { between } s_{1} \text { and } s_{3} \\
\text { between } s_{5} \text { and } s_{6} & \text { at most } s_{1} & \text { between } s_{5} \text { and } s_{7} & s_{4}
\end{array}\right]
\end{aligned}
$$

$$
\begin{aligned}
\mathcal{P}^{3} & =\left[\begin{array}{cccc}
s_{4} & \text { at least } s_{5} & \text { between } s_{5} \text { and } s_{6} & \text { between } s_{1} \text { and } s_{3} \\
\text { at most } s_{3} & s_{4} & \text { between } s_{6} \text { and } s_{7} & s_{0} \\
\text { between } s_{2} \text { and } s_{3} & \text { between } s_{1} \text { and } s_{2} & s_{4} & s_{2} \\
\text { between } s_{5} \text { and } s_{7} & s_{8} & s_{6} & s_{4} \\
s_{4} & s_{5} & \text { between } s_{2} \text { and } s_{3} & \text { at least } s_{5} \\
s_{3} & s_{4} & \text { at most } s_{3} & \text { at least } s_{7} \\
\mathcal{P}^{4} & =\left[\begin{array}{cccc}
s_{4} & s_{7}
\end{array}\right] \\
\text { between } s_{5} \text { and } s_{6} & \text { at least } s_{5} & s_{1} & s_{4}
\end{array}\right]
\end{aligned}
$$$$
\mathcal{P}^{5}=\left[\begin{array}{cccc}
s_{4} & \text { at least } s_{7} & \text { at most } s_{2} & s_{4} \\
\text { at most } s_{1} & s_{4} & \text { at least } s_{5} & s_{8} \\
\text { at least } s_{6} & \text { at most } s_{3} & s_{4} & \text { between } s_{5} \text { and } s_{7} \\
s_{4} & s_{0} & \text { between } s_{1} \text { and } s_{3} & s_{4}
\end{array}\right]
$$

- $\quad$ Step 2: to obtain the induced similarity order of $i^{\text {th }}$ row of $\mathcal{P}^{k}, k=1,2,3,4,5$.

Using the first row of $\mathcal{P}^{1}$ as an example to illustrate how to obtain the induced similarity order.

$$
\mathcal{P}^{1}=\left[\begin{array}{cccc}
S_{4} & \text { at most } S_{3} & \text { between } S_{6} \text { and } S_{7} & S_{1} \\
\text { at least } S_{5} & S_{4} & \text { at least } S_{6} & \text { at least } S_{6} \\
\text { between } S_{1} \text { and } S_{2} & \text { at most } S_{2} & S_{4} & \text { between } S_{2} \text { and } S_{3} \\
S_{7} & \text { at most } S_{2} & \text { between } S_{5} \text { and } S_{6} & S_{4}
\end{array}\right]
$$

The first row of $\mathcal{P}^{1}$ is $\left\{p_{11}^{1}, p_{12}^{1}, p_{13}^{1}, p_{14}^{1}\right\}=\left\{S_{4}\right.$, at most $S_{3}$, between $S_{6}$ and $\left.S_{7}, S_{1}\right\}$ and the overall distance measure for the $1^{\text {st }}$ row of the matrix $\mathcal{P}^{1}$ is shown in Table 2 . Upon Table 2 and the increasing lexicographic order of the pair $\left(\operatorname{Sum}\left(p_{1 j}^{1}\right), \phi\left(p_{1 j}^{1}\right)\right), j=$ $1,2,3,4$, we obtain $\operatorname{Sum}\left(p_{13}^{1}\right)<\operatorname{Sum}\left(p_{14}^{1}\right)<\operatorname{Sum}\left(p_{12}^{1}\right)=\operatorname{Sum}\left(p_{11}^{1}\right)$ and $\phi\left(p_{12}^{1}\right)=\frac{3}{2}<$ $\phi\left(p_{11}^{1}\right)=4$.

Therefore, the induced similarity order

$$
p_{13}^{1} \prec p_{14}^{1} \prec p_{12}^{1} \prec p_{11}^{1} .
$$

Similarly, we obtain the induced similarity order of each row of the $\mathcal{P}^{1}$ as follows:

$$
p_{13}^{1} \prec p_{14}^{1} \prec p_{12}^{1} \prec p_{11}^{1} ; p_{22}^{1} \prec p_{24}^{1}=p_{23}^{1} \prec p_{21}^{1} ; p_{33}^{1} \prec p_{32}^{1} \prec p_{31}^{1} \prec p_{34}^{1} ; p_{42}^{1} \prec p_{41}^{1} \prec p_{43}^{1} \prec p_{44}^{1} ;
$$


Hence, we obtain the ordered $\mathcal{P}^{1}$ as $\mathcal{P}_{\text {ordered }}^{1}$ as the following one:

$$
\mathcal{P}_{\text {ordered }}^{1}=\left[\begin{array}{llll}
p_{13}^{1} & p_{14}^{1} & p_{12}^{1} & p_{11}^{1} \\
p_{22}^{1} & p_{24}^{1} & p_{23}^{1} & p_{21}^{1} \\
p_{33}^{1} & p_{32}^{1} & p_{31}^{1} & p_{34}^{1} \\
p_{42}^{1} & p_{41}^{1} & p_{43}^{1} & p_{44}^{1}
\end{array}\right]
$$

- $\quad$ Step 3: using the ELICIT-t1-IOWA operator to aggregate the ordered matrix $\mathcal{P}^{1}$ with the assigned finite fuzzy weighting vector is $\widetilde{W}=\left\{\widetilde{w}_{1}, \widetilde{w}_{2}, \widetilde{w}_{3}, \widetilde{w}_{4}\right\}$, such that the interval weight $\widetilde{w}_{i}$ is computed by the type-2 linguistic quantifier "most" introduced in [32] , then

$$
\widetilde{w}_{1}=[0,0], \widetilde{w}_{2}=[0,0.4], \widetilde{w}_{3}=[0.433,0.9], \widetilde{w}_{4}=[0.1,0.167] .
$$

Applying the ELICIT-t1-IOWA operator with fuzzy weight to the ordered $\mathcal{P}^{1}$ in order to obtain the following results:

$$
\begin{aligned}
a_{1}^{1} & =\frac{\widetilde{w}_{1} \otimes \zeta^{-1}\left(p_{13}^{1}\right)+\widetilde{w}_{2} \otimes \zeta^{-1}\left(p_{14}^{1}\right)+\widetilde{w}_{3} \otimes \zeta^{-1}\left(p_{12}^{1}\right)+\widetilde{w}_{4} \otimes \zeta^{-1}\left(p_{11}^{1}\right)}{\Sigma_{j=1}^{4} \widetilde{w}_{j}} \\
& =\frac{\widetilde{w}_{1} \otimes T(0.625,0.75,0.875,1)+\widetilde{w}_{2} \otimes T(0,0.125,0.25)+\widetilde{w}_{3} \otimes T(0,0,0.375,0.5)+\widetilde{w}_{4} \otimes T(0.375,0.5,0.625)}{\Sigma_{j=1}^{4} \widetilde{w}_{j}} \\
& =T(0.026786,0.050000,0.40979,0.53479) ; \\
a_{2}^{1} & =\frac{\widetilde{w}_{1} \otimes \zeta^{-1}\left(p_{22}^{1}\right)+\widetilde{w}_{2} \otimes \zeta^{-1}\left(p_{24}^{1}\right)+\widetilde{w}_{3} \otimes \zeta^{-1}\left(p_{23}^{1}\right)+\widetilde{w}_{4} \otimes \zeta^{-1}\left(p_{21}^{1}\right)}{\sum_{j=1}^{4} \widetilde{w}_{j}} \\
& =T(0.59021,0.71521,1,1) ; \\
a_{3}^{1} & =\frac{\widetilde{w}_{1} \otimes \zeta^{-1}\left(p_{33}^{1}\right)+\widetilde{w}_{2} \otimes \zeta^{-1}\left(p_{32}^{1}\right)+\widetilde{w}_{3} \otimes \zeta^{-1}\left(p_{31}^{1}\right)+\widetilde{w}_{4} \otimes \zeta^{-1}\left(p_{34}^{1}\right)}{\Sigma_{j=1}^{4} \widetilde{w}_{j}} \\
& =T(0.0089286,0.084807,0.28479,0.40979) ; \\
a_{4}^{1} & =\frac{\widetilde{w}_{1} \otimes \zeta^{-1}\left(p_{42}^{1}\right)+\widetilde{w} \otimes \zeta^{-1}\left(p_{41}^{1}\right)+\widetilde{w}_{4} \otimes \zeta^{-1}\left(p_{43}^{1}\right)+\widetilde{w} \otimes \zeta^{-1}\left(p_{44}^{1}\right)}{\Sigma_{j=1}^{4} \widetilde{w}_{j}} \\
& =T(0.46521,0.59021,0.77680,0.90180) ;
\end{aligned}
$$

Hence, $\mathcal{M}^{1}=\left[\begin{array}{l}a_{1}^{1} \\ a_{2}^{1} \\ a_{3}^{1} \\ a_{4}^{1}\end{array}\right]=\left[\begin{array}{c}T(0.026786,0.050000,0.40979,0.53479) \\ T(0.59021,0.71521,1,1) \\ T(0.0089286,0.084807,0.28479,0.40979) \\ T(0.46521,0.59021,0.77680,0.90180)\end{array}\right]$.

Similarly, we will obtain the ordered $\mathcal{P}^{k}$ and the aggregated results $\mathcal{M}^{k}$ for all $k=$ $2,3,4,5$, as follows:

$$
\begin{aligned}
\mathcal{P}_{\text {ordered }}^{2}= & {\left[\begin{array}{llll}
p_{14}^{2} & p_{13}^{2} & p_{11}^{2} & p_{12}^{2} \\
p_{24}^{2} & p_{23}^{2} & p_{21}^{2} & p_{22}^{2} \\
p_{32}^{2} & p_{31}^{2} & p_{34}^{2} & p_{33}^{2} \\
p_{42}^{2} & p_{43}^{2} & p_{44}^{2} & p_{41}^{2}
\end{array}\right] ; \mathcal{P}_{\text {ordered }}^{3}=\left[\begin{array}{llll}
p_{14}^{3} & p_{12}^{3} & p_{11}^{3} & p_{13}^{3} \\
p_{23}^{3} & p_{24}^{3} & p_{21}^{3} & p_{22}^{3} \\
p_{32}^{3} & p_{33}^{3} & p_{31}^{3} & p_{34}^{3} \\
p_{44}^{3} & p_{42}^{3} & p_{43}^{3} & p_{41}^{3}
\end{array}\right] ; } \\
\mathcal{P}_{\text {ordered }}^{4}= & {\left[\begin{array}{llll}
p_{13}^{4} & p_{14}^{4} & p_{11}^{4} & p_{12}^{4} \\
p_{24}^{4} & p_{23}^{4} & p_{21}^{4} & p_{22}^{4} \\
p_{33}^{4} & p_{34}^{4} & p_{31}^{4} & p_{32}^{4} \\
p_{44}^{4} & p_{42}^{4} & p_{43}^{4} & p_{41}^{4}
\end{array}\right] ; \mathcal{P}_{\text {ordered }}^{5}=\left[\begin{array}{llll}
p_{12}^{5} & p_{13}^{5} & p_{14}^{5} & p_{11}^{5} \\
p_{21}^{5} & p_{24}^{5} & p_{22}^{5} & p_{23}^{5} \\
p_{32}^{5} & p_{31}^{5} & p_{33}^{5} & p_{34}^{5} \\
p_{42}^{5} & p_{43}^{5} & p_{44}^{5} & p_{41}^{5}
\end{array}\right] }
\end{aligned}
$$




$$
\begin{gathered}
\mathcal{M}^{2}=\left[\begin{array}{l}
a_{1}^{2} \\
a_{2}^{2} \\
a_{3}^{2} \\
a_{4}^{2}
\end{array}\right]=\left[\begin{array}{c}
T(0.38750,0.51250,0.67417,0.79917) \\
T(0.15622,0.22763,0.40979,0.53479) \\
T(0.026786,0.15179,0.40979,0.53479) \\
T(0.38750,0.51250,0.69175,0.81675)
\end{array}\right] ; \\
\mathcal{M}^{3}=\left[\begin{array}{l}
a_{1}^{3} \\
a_{2}^{3} \\
a_{3}^{3} \\
a_{4}^{3}
\end{array}\right]=\left[\begin{array}{c}
T(0.38750,0.51250,0.74175,0.81675) \\
T(0.026786,0.035714,0.40979,0.53479) \\
T(0.12500,0.25000,0.41519,0.54019) \\
T(0.12500,0.25000,0.41519,0.54019)
\end{array}\right] ; \\
\mathcal{M}^{4}=\left[\begin{array}{l}
a_{1}^{4} \\
a_{2}^{4} \\
a_{3}^{4} \\
a_{4}^{4}
\end{array}\right]=\left[\begin{array}{c}
T(0.38750,0.51250,0.72776,0.79917) \\
T(0.15622,0.22763,0.40979,0.53479) \\
T(0.50000,0.62500,0.84175,0.94588) \\
T(0.16237,0.23738,0.46250,0.58750)
\end{array}\right] ; \\
\mathcal{M}^{5}=\left[\begin{array}{l}
T(0.21423,0.28564,0.50000,0.62500) \\
T(0.38750,0.51250,0.78350,0.83763) \\
T(0.38750,0.51250,0.76262,0.83763) \\
a_{2}^{5} \\
a_{3}^{5} \\
a_{4}^{5}
\end{array}\right]=\left[\begin{array}{l}
T \\
5
\end{array}\right]
\end{gathered}
$$

- $\quad$ Step 4: using the ELICIT-I-IOWA operator to aggregate $\mathcal{M}^{k}$ with linguistic quantifier $Q$ "most", then the BUM function $Q(r)$ is $Q(r)=\left\{\begin{array}{c}0, \text { if } 0 \leq r<0.3 \\ 2 r-0.6, \text { if } 0.3 \leq r \leq 0.8 \\ 1, \text { if } 0.8<r \leq 1\end{array}\right.$.

Suppose that the experts associated with the degree of prestige that is a proportion of $[0,1]$ are recorded as

$$
\left\{\left\langle e_{k}, \alpha_{k}\right\rangle\right\}_{k=1}^{5}=\left\{\left\langle e_{1}, 0.6\right\rangle,\left\langle e_{2}, 1\right\rangle,\left\langle e_{3}, 0.5\right\rangle,\left\langle e_{4}, 0.9\right\rangle,\left\langle e_{5}, 0.7\right\rangle\right\}
$$

We will obtain the reorder of $\left\{\left\langle e_{k}, \alpha_{k}\right\rangle\right\}_{k=1}^{5}$ and the weight as

$$
\begin{gathered}
\left\{\left\langle e_{2}, 1\right\rangle,\left\langle e_{4}, 0.9\right\rangle,\left\langle e_{5}, 0.7\right\rangle,\left\langle e_{1}, 0.6\right\rangle,\left\langle e_{3}, 0.5\right\rangle\right\} \\
\omega_{1}=0, \omega_{2}=0.42703, \omega_{3}=0.37838, \omega_{4}=0.19459, \omega_{5}=0
\end{gathered}
$$

Applying the ELICIT-I-IOWA operator to $\left\{\left\langle e_{k}, \alpha_{k}\right\rangle\right\}_{k=1}^{5}$, we will obtain the result

$$
\mathcal{R}=\Sigma_{k=1}^{5} \omega_{k} \times \mathcal{M}^{\sigma(k)}=\left[\begin{array}{l}
\beta_{1} \\
\beta_{2} \\
\beta_{3} \\
\beta_{4}
\end{array}\right]=\left[\begin{array}{c}
T(0.25175,0.33666,0.57971,0.68182) \\
T(0.32818,0.43030,0.66604,0.73990) \\
T(0.36187,0.47732,0.70343,0.80060) \\
T(0.24092,0.34458,0.53785,0.66285)
\end{array}\right]
$$

where $\beta_{i}=\Sigma_{k=1}^{5} \omega_{k} \times a_{i}^{\sigma(k)}$ for all $i=1,2,3,4$.

- Step 5: ranking $\beta_{i}$ to select top one alternative as a solution to this problem and then complete the ELICIT-CW scheme [15] in order to obtain the final ELICIT expressions.

Because $\beta_{i}$ is a trapezoidal fuzzy number with no inherent order, we need to choose a method for defining a comparison operator between fuzzy numbers. Here, we choose the method, the so-called "Magnitude" function, provided by Abbasbandy and Hajjari [45], because, the larger magnitude, the larger the fuzzy number. Following the definition of the "Magnitude", $\operatorname{Mag}(\cdot)$, we obtain the results: $\operatorname{Mag}\left(\beta_{1}\right)=0.45962 ; \operatorname{Mag}\left(\beta_{2}\right)=$ $0.54582 ; \operatorname{Mag}\left(\beta_{3}\right)=0.58885 ; \operatorname{Mag}\left(\beta_{4}\right)=0.44299$, then $\beta_{3}>\beta_{2}>\beta_{1}>\beta_{4}$. Hence, the ranking of alternatives is $a_{4} \prec a_{1} \prec a_{2} \prec a_{3}$, so that the alternative $a_{3}$ is the top one as a solution to this problem. Table 3 represents the overall result upon "majority opinion". 
Table 2. The overall distance measure for the $1^{\text {st }}$ row of the matrix $\mathcal{P}^{1}$.

\begin{tabular}{cccccc}
\hline $\boldsymbol{i}=\mathbf{1}$ & $\boldsymbol{p}_{\mathbf{1 1}}^{\mathbf{1}}$ & $\boldsymbol{p}_{\mathbf{1 2}}^{\mathbf{1}}$ & $\boldsymbol{p}_{\mathbf{1 3}}^{\mathbf{1}}$ & $\boldsymbol{p}_{\mathbf{1 4}}^{\mathbf{1}}$ & Sum \\
\hline$p_{11}^{1}$ & 0 & $\frac{5}{2}$ & $\frac{5}{2}$ & $\frac{7}{2}$ & $-\frac{17}{2}$ \\
\hline$p_{12}^{1}$ & $\frac{5}{2}$ & 0 & 5 & 1 & $-\frac{17}{2}$ \\
\hline$p_{13}^{1}$ & $\frac{5}{2}$ & 5 & 0 & 6 & $-\frac{27}{2}$ \\
\hline$p_{14}^{1}$ & $\frac{7}{2}$ & 2 & 6 & 0 & $-\frac{21}{2}$ \\
\hline
\end{tabular}

Table 3. The overall result upon "majority opinion".

\begin{tabular}{cll}
\hline Alternative & \multicolumn{1}{c}{$\beta_{\boldsymbol{i}}$} & $\zeta\left(\beta_{i}\right)$ \\
\hline$a_{1}$ & $T(0.25175,0.33666,0.57971,0.68182)$ & between $\left(S_{3},-0.31\right)^{0.04}$ and $\left(S_{5},-0.36\right)^{-0.023}$ \\
$a_{2}$ & $T(0.32818,0.43030,0.66604,0.73990)$ & between $\left(S_{3}, 0.44\right)^{0.023}$ and $\left(S_{5}, 0.33\right)^{-0.051}$ \\
$a_{3}$ & $T(0.36187,0.47732,0.70343,0.80060)$ & between $\left(S_{4},-0.18\right)^{0.009}$ and $\left(S_{6},-0.37\right)^{-0.028}$ \\
$a_{4}$ & $T(0.24092,0.34458,0.53785,0.66285)$ & between $\left(S_{3},-0.24\right)^{0.021}$ and $\left(S_{4}, 0.3\right)$ \\
\hline
\end{tabular}

\subsection{Comparative Analysis}

This section provides a comparative analysis that includes two different views in order to show the performance of the previous GDM majority-driven solving process driven by ELICIT-t1-OWA and ELICIT-I-IOWA:

1. In the previous resolution scheme, the ELICIT-t1-IOWA operator with fuzzy interval weight is only computed by the type-2 linguistic quantifier "most" in order to aggregate the most similar opinions. However, the weights of the ELICIT-I-IOWA operator can be obtained from different linguistic quantifiers according to the aggregated opinion chased. Therefore, we will apply various linguistic quantifiers to compute the weights ELICIT-I-IOWA operator combining with the same ELICIT-t1-IOWA operator and comparing the results.

2. A second view for comparison is related to previous operators in order to aggregate ELICIT information. However, so far, just two of them have been introduced; namely, the fuzzy arithmetic mean [15] and the Bonferroni mean aggregation operator [21]. Because of the type of problem that we are dealing with, the Bonferroni mean operator cannot be used because there is no interaction among the criteria. Therefore, we propose replacing the ELICIT-t1-IOWA operator with the fuzzy arithmetic mean in the majority-driven GDM processes to combine it with the ELICIT-I-IOWA operator in the previous GDM problem. Additionally, compare the final ranking results with the ones from the previous subsection.

6.3.1. A Majority-Driven Solving Process Driven by Elicit-T1-Owa and Elicit-I-Iowa with Different Linguistic Quantifiers

It is noticeable that the aggregation phase of the majority-driven GDM process consists of the ELICIT-t1-IOWA operator to each ELICIT preference relation matrix $\mathcal{P}^{k}$ for each expert $e_{k}$ to obtain $\mathcal{M}^{k}$ and the ELICIT-I-IOWA operator to the all matrix $\mathcal{M}^{k}$ with a degree of prestige of expert $e_{k}$ into an overall result $\mathcal{R}$. The ELICIT-t1-IOWA operator and the ELICIT-I-IOWA operator both use the linguistic quantifier "most" in order to consider "majority opinion". For the sake of clarity, when considering that the type-2 linguistic quantifier only uses the interval-valued type-2 linguistic quantifier "most" [32] induced the interval weight; therefore, we will compare the results that were obtained in the previous example with the BUM function $Q(r)$ that represents the linguistic quantifier "at least half" with $\alpha=0, \beta=0.5$, linguistic quantifier "as many as possible" with $\alpha=0.5, \beta=0.8$ and linguistic quantifier "mean" with $\alpha=0, \beta=1$. 
We apply the ELICIT-I-IOWA operator with different linguistic quantifiers to $\mathcal{M}^{k}$, $k=1,2,3,4,5$.

1. While using linguistic quantifier "at least half" with $\alpha=0, \beta=0.5$.

In this case, we obtain the weights

$$
\omega_{1}=0.54054, \omega_{2}=0.45946, \omega_{3}=0, \omega_{4}=0, \omega_{5}=0
$$

Applying the ELICIT-I-IOWA operator to $\left\{\left\langle e_{k}, \alpha_{k}\right\rangle\right\}_{k=1}^{5}$ in order to obtain the result as follows:

$$
\mathcal{R}_{\text {Case } 1}=\left[\begin{array}{l}
\beta_{1} \\
\beta_{2} \\
\beta_{3} \\
\beta_{4}
\end{array}\right]=\left[\begin{array}{l}
T(0.38750,0.51250,0.69879,0.79917) \\
T(0.15622,0.22763,0.40979,0.53479) \\
T(0.24421,0.36921,0.60826,0.72367) \\
T(0.28406,0.38609,0.58642,0.71142)
\end{array}\right]
$$

Implementing the "Magnitude" function $\operatorname{Mag}(\cdot)$, we obtain

$\operatorname{Mag}\left(\beta_{1}\right)=0.60359 ; \operatorname{Mag}\left(\beta_{2}\right)=0.32318 ; \operatorname{Mag}\left(\beta_{3}\right)=0.48794 ;$ and, $\operatorname{Mag}\left(\beta_{4}\right)=0.48817$.

Hence, the ranking of alternatives is $a_{2} \prec a_{3} \prec a_{4} \prec a_{1}$.

2. Using linguistic quantifier "as many as possible" with $\alpha=0.5, \beta=0.8$.

In this case we obtain the weights

$$
\omega_{1}=0, \omega_{2}=0.045045, \omega_{3}=0.63063, \omega_{4}=0.32432, \omega_{5}=0
$$

Applying the ELICIT-I-IOWA operator to $\left\{\left\langle e_{k}, \alpha_{k}\right\rangle\right\}_{k=1}^{5}$ to obtain the result as follows:

$$
\mathcal{R}_{\text {Case } 2}=\left[\begin{array}{l}
\beta_{1} \\
\beta_{2} \\
\beta_{3} \\
\beta_{4}
\end{array}\right]=\left[\begin{array}{c}
T(0.16124,0.21943,0.48100,0.60359) \\
T(0.44282,0.56541,0.83688,0.87664) \\
T(0.26979,0.37886,0.61121,0.70374) \\
T(0.29329,0.41604,0.58808,0.71308)
\end{array}\right]
$$

Implementing the "Magnitude" function $\operatorname{Mag}(\cdot)$, we obtain

$$
\operatorname{Mag}\left(\beta_{1}\right)=0.35558 ; \operatorname{Mag}\left(\beta_{2}\right)=0.69424 ; \operatorname{Mag}\left(\beta_{3}\right)=0.49366 ; \operatorname{Mag}\left(\beta_{4}\right)=0.50225 \operatorname{~}
$$

Hence, the ranking of alternatives is $a_{1} \prec a_{3} \prec a_{4} \prec a_{2}$.

3. Using linguistic quantifier "mean"with $\alpha=0, \beta=1$.

In this case, we obtain the weights

$$
\omega_{1}=0.27027, \omega_{2}=0.24324, \omega_{3}=0.18919, \omega_{4}=0.16216, \omega_{5}=0.13513
$$

Applying the ELICIT-I-IOWA operator to $\left\{\left\langle e_{k}, \alpha_{k}\right\rangle\right\}_{k=1}^{5}$ to obtain the result as follows:

$$
\mathcal{R}_{\text {Case } 3}=\left[\begin{array}{l}
\beta_{1} \\
\beta_{2} \\
\beta_{3} \\
\beta_{4}
\end{array}\right]=\left[\begin{array}{c}
T(0.29622,0.39458,0.62051,0.72571) \\
T(0.25286,0.33465,0.57620,0.66752) \\
T(0.22051,0.33754,0.56207,0.67253) \\
T(0.27708,0.38992,0.57612,0.70112)
\end{array}\right]
$$

Implementing the "Magnitude" function $\operatorname{Mag}(\cdot)$, we obtain

$$
\operatorname{Mag}\left(\beta_{1}\right)=0.50811 ; \operatorname{Mag}\left(\beta_{2}\right)=0.45622 ; \operatorname{Mag}\left(\beta_{3}\right)=0.44926 ; \operatorname{Mag}\left(\beta_{4}\right)=0.48403 \text {. }
$$


Hence, the ranking of alternatives is $a_{3} \prec a_{2} \prec a_{4} \prec a_{1}$.

We can observe that the ranking changes according to the different linguistic quantifiers, which means that we then add up the prestige of the experts until we obtain different degrees of prestige. Based on the majority-driven GDM approach, we know that the result of this method is reliable.

Finally, the aggregated results $\mathcal{R}$ are re-translated into ELICIT expressions by function $\zeta$ [15]; for experts, these results are very intuitive (see Table 4).

Table 4. The overall result with ELICIT expressions.

\begin{tabular}{cccc}
\hline & Aggregated Results $\mathcal{R}$ & ELICIT Expression $\zeta(\mathcal{R})$ & Ranking Result \\
\hline "most" & $T(0.25175,0.33666,0.57971,0.68182)$ & bt $\left(S_{3},-0.31\right)^{0.04}$ and $\left(S_{5},-0.36\right)^{-0.023}$ & $a_{4} \prec a_{1} \prec a_{2} \prec a_{3}$ \\
& $T(0.32818,0.43030,0.66604,0.73990)$ & bt $\left(S_{3}, 0.44\right)^{0.023}$ and $\left(S_{5}, 0.33\right)^{-0.051}$ & \\
$T(0.36187,0.47732,0.70343,0.80060)$ & bt $\left(S_{4},-0.18\right)^{0.009}$ and $\left(S_{6},-0.37\right)^{-0.028}$ & \\
$T(0.24092,0.34458,0.53785,0.66285)$ & bt $\left(S_{3},-0.24\right)^{0.021}$ and $\left(S_{4}, 0.3\right)$ & \\
ine Case 1 & $T(0.38750,0.51250,0.69879,0.79917)$ & bt $\left(S_{4}, 0.1\right)$ and $\left(S_{6},-0.41\right)^{-0.025}$ & $a_{2} \prec a_{3} \prec a_{4} \prec a_{1}$ \\
& $T(0.15622,0.22763,0.40979,0.53479)$ & bt $\left(S_{2},-0.18\right)^{0.054}$ and $\left(S_{3}, 0.28\right)$ & \\
& $T(0.24421,0.36921,0.60826,0.72367)$ & bt $\left(S_{3},-0.05\right)$ and $\left(S_{5},-0.13\right)^{-0.01}$ & \\
& $T(0.28406,0.38609,0.58642,0.71142)$ & bt $\left(S_{3}, 0.09\right)^{0.023}$ and $\left(S_{5},-0.31\right)$ & \\
ine Case 2 & $T(0.16124,0.21943,0.48100,0.60359)$ & bt $\left(S_{2},-0.24\right)^{0.066}$ and $\left(S_{4},-0.15\right)^{-0.003}$ & $a_{1} \prec a_{3} \prec a_{4} \prec a_{2}$ \\
& $T(0.44282,0.56541,0.83688,0.87664)$ & bt $\left(S_{5},-0.48\right)^{0.003}$ and $\left(S_{7},-0.3\right)^{-0.086}$ & \\
& $T(0.26979,0.37886,0.61121,0.70374)$ & bt $\left(S_{3}, 0.03\right)^{0.016}$ and $\left(S_{5},-0.11\right)^{-0.033}$ & \\
& $T(0.29329,0.41604,0.58808,0.71308)$ & bt $\left(S_{3}, 0.33\right)^{0.002}$ and $\left(S_{5},-0.3\right)^{0.001}$ & \\
ine Case 3 & $T(0.29622,0.39458,0.62051,0.72571)$ & bt $\left(S_{3}, 0.16\right)^{0.026}$ and $\left(S_{5},-0.04\right)^{-0.019}$ & $a_{3} \prec a_{2} \prec a_{4} \prec a_{1}$ \\
& $T(0.25286,0.33465,0.57620,0.66752)$ & bt $\left(S_{3},-0.32\right)^{0.043}$ and $\left(S_{5},-0.39\right)^{-0.034}$ & \\
$T(0.22051,0.33754,0.56207,0.67253)$ & bt $\left(S_{3},-0.3\right)^{0.008}$ and $\left(S_{4}, 0.5\right)^{-0.015}$ & \\
& $T(0.27708,0.38992,0.57612,0.70112)$ & bt $\left(S_{3}, 0.12\right)^{0.012}$ and $\left(S_{5},-0.39\right)$ & \\
\hline
\end{tabular}

In Table 4 "bt" stands for "between".

\subsubsection{A Solving Process Driven by Fuzzy Arithmetic Mean and The Elicit-I-Owa}

If we consider that the order inducing variable is the same as the argument and assigned with the weight $\left(\frac{1}{n}, \cdots, \frac{1}{n}\right)$, the fuzzy arithmetic mean operator is a special case of the IOWA operator, thus we will replace the ELICIT-t1-IOWA operator with the fuzzy arithmetic mean in the current proposal, the ELICIT-I-IOWA operator used will be guided by the previous linguistic quantifiers that were used in previous sections. Finally, we will compare the final ranking results between the ELICIT-t1-IOWA operator and fuzzy arithmetic mean operator, with both of them combining the same ELICIT-I-IOWA operator in the majority-driven process.

Inspired by the ELICIT-IOWA, we will obtain the formula of the application of the fuzzy arithmetic mean operator to ELICIT information as the following one:

$$
\mathbf{F}_{\text {mean }}\left(x_{1}, \cdots, x_{n}\right)=\zeta\left(\frac{1}{n} \times \sum_{i=1}^{n} \zeta^{-1}\left(x_{i}\right)\right)
$$

1. Using the same example in Section 6.1, applying the Equation (40) on the preference relation matrix $\mathcal{P}^{k}, k=1,2,3,4,5$, we will obtain the result as

$$
\mathcal{A}^{1}=\left[\begin{array}{l}
a_{1}^{1} \\
a_{2}^{1} \\
a_{3}^{1} \\
a_{4}^{1}
\end{array}\right]=\left[\begin{array}{c}
T(0.25000,0.34375,0.46875,0.59375) \\
T(0.53125,0.65625,0.87500,0.90625) \\
T(0.12500,0.21875,0.34375,0.46875) \\
T(0.40625,0.50000,0.59375,0.71875)
\end{array}\right] ;
$$




$$
\begin{aligned}
& \mathcal{A}^{2}=\left[\begin{array}{l}
a_{1}^{2} \\
a_{2}^{2} \\
a_{3}^{2} \\
a_{4}^{2}
\end{array}\right]=\left[\begin{array}{l}
T(0.40625,0.53125,0.59375,0.71875) \\
T(0.34375,0.43750,0.50000,0.59375) \\
T(0.28125,0.40625,0.53125,0.62500) \\
T(0.34375,0.43750,0.56250,0.68750)
\end{array}\right] ; \\
& \mathcal{A}^{3}=\left[\begin{array}{l}
a_{1}^{3} \\
a_{2}^{3} \\
a_{3}^{3} \\
a_{4}^{3}
\end{array}\right]=\left[\begin{array}{l}
T(0.34375,0.46875,0.65625,0.75000) \\
T(0.25000,0.31250,0.43750,0.56250) \\
T(0.15625,0.28125,0.34375,0.46875) \\
T(0.34375,0.43750,0.56250,0.68750)
\end{array}\right] ; \\
& \mathcal{A}^{4}=\left[\begin{array}{l}
a_{1}^{4} \\
a_{2}^{4} \\
a_{3}^{4} \\
a_{4}^{4}
\end{array}\right]=\left[\begin{array}{l}
T(0.37500,0.50000,0.62500,0.71875) \\
T(0.34375,0.43750,0.56250,0.65625) \\
T(0.53125,0.65625,0.78125,0.87500) \\
T(0.18750,0.28125,0.31250,0.43750)
\end{array}\right] ; \\
& \mathcal{A}^{5}=\left[\begin{array}{l}
a_{1}^{5} \\
a_{2}^{5} \\
a_{3}^{5} \\
a_{4}^{5}
\end{array}\right]=\left[\begin{array}{c}
T([0.37500,0.46875,0.56250,0.65625) \\
T(0.43750,0.53125,0.65625,0.71875) \\
T(0.37500,0.46875,0.68750,0.78125) \\
T(0.18750,0.28125,0.34375,0.46875)
\end{array}\right] ;
\end{aligned}
$$

2. Utilizing various linguistic quantifiers of the ELICIT-I-IOWA operator on the matrix $\mathcal{A}^{k}, k=1,2,3,4,5$ : linguistic quantifier "most" with $\alpha=0.3, \beta=0.8$, linguistic quantifier "at least half" with $\alpha=0, \beta=0.5$, linguistic quantifier "as many as possible" with $\alpha=0.5, \beta=0.8$ and linguistic quantifier "mean" with $\alpha=0, \beta=1$. Therefore, the process is similar to the previous section; we will skip the process and directly give the following results:

$$
\mathcal{R}_{\text {most }}=\left[\begin{array}{c}
\beta_{1} \\
\beta_{2} \\
\beta_{3} \\
\beta_{4}
\end{array}\right]=\left[\begin{array}{c}
T(0.35068,0.45777,0.57095,0.67078) \\
T(0.41571,0.51554,0.65878,0.72855) \\
T(0.39308,0.50017,0.66064,0.76047) \\
T(0.23007,0.32382,0.37905,0.50405)
\end{array}\right]
$$

Implementing the "Magnitude" function $\operatorname{Mag}(\cdot)$, we will obtain

$$
\operatorname{Mag}\left(\beta_{1}\right)=0.51375 ; \operatorname{Mag}\left(\beta_{2}\right)=0.58466 ; \operatorname{Mag}\left(\beta_{3}\right)=0.57980 ; \operatorname{Mag}\left(\beta_{4}\right)=0.35404 \text {. }
$$

Hence, the ranking of alternatives is $a_{4} \prec a_{1} \prec a_{3} \prec a_{2}$.

$$
\mathcal{R}_{\text {at least half }}=\left[\begin{array}{l}
\beta_{1} \\
\beta_{2} \\
\beta_{3} \\
\beta_{4}
\end{array}\right]=\left[\begin{array}{c}
T(0.39189,0.51689,0.60811,0.71875) \\
T(0.34375,0.43750,0.52872,0.62247) \\
T(0.39611,0.52111,0.64611,0.73986) \\
T(0.27196,0.36571,0.44764,0.57264)
\end{array}\right]
$$

Implementing the "Magnitude" function $\operatorname{Mag}(\cdot)$, we will obtain

$$
\operatorname{Mag}\left(\beta_{1}\right)=0.56130 ; \operatorname{Mag}\left(\beta_{2}\right)=0.48311 ; \operatorname{Mag}\left(\beta_{3}\right)=0.58101 ; \operatorname{Mag}\left(\beta_{4}\right)=0.40928 \text {. }
$$

Hence, the ranking of alternatives is $a_{4} \prec a_{2} \prec a_{1} \prec a_{3}$.

$$
\mathcal{R}_{\text {as many as possible }}=\left[\begin{array}{l}
\beta_{1} \\
\beta_{2} \\
\beta_{3} \\
\beta_{4}
\end{array}\right]=\left[\begin{array}{c}
T(0.33446,0.42962,0.53491,0.63879) \\
T(0.46368,0.56756,0.72297,0.77674) \\
T(0.30096,0.39611,0.58023,0.68412) \\
T(0.25844,0.35219,0.42342,0.54842)
\end{array}\right]
$$

Implementing the "Magnitude" function $\operatorname{Mag}(\cdot)$, we will obtain

$$
\operatorname{Mag}\left(\beta_{1}\right)=0.48299 ; \operatorname{Mag}\left(\beta_{2}\right)=0.64109 ; \operatorname{Mag}\left(\beta_{3}\right)=0.48890 ; \operatorname{Mag}\left(\beta_{4}\right)=0.39041 \text {. }
$$


Hence, the ranking of alternatives is $a_{4} \prec a_{1} \prec a_{3} \prec a_{2}$.

$$
\mathcal{R}_{\text {mean }}=\left[\begin{array}{l}
\beta_{1} \\
\beta_{2} \\
\beta_{3} \\
\beta_{4}
\end{array}\right]=\left[\begin{array}{c}
T(0.35895,0.47297,0.58361,0.69087) \\
T(0.37922,0.47381,0.59712,0.67905) \\
T(0.31756,0.43158,0.56587,0.66891) \\
T(0.28631,0.38006,0.46537,0.59037)
\end{array}\right]
$$

Implementing the "Magnitude" function $\operatorname{Mag}(\cdot)$, we will obtain

$$
\operatorname{Mag}\left(\beta_{1}\right)=0.52773 ; \operatorname{Mag}\left(\beta_{2}\right)=0.53441 ; \operatorname{Mag}\left(\beta_{3}\right)=0.49781 ; \operatorname{Mag}\left(\beta_{4}\right)=0.42532 .
$$

Thence, the ranking of alternatives is $a_{4} \prec a_{3} \prec a_{1} \prec a_{2}$.

3. Comparing the final ranking results between the fuzzy arithmetic mean operator and ELICIT-t1-IOWA operator combining various ELICIT-I-IOWA operator. Looking at Table 5, it can be observed that the overall results of applying the fuzzy arithmetic mean operator to the previous example are quite steady disregarding the aggregation of the ELICIT-I-IOWA operator and the linguistic quantifiers that are used by it. However, the ELICIT-t1-IOWA operator is much more sensitive to different situations that are modeled by the linguistic quantifiers used in the ELICIT-I-IOWA operator. This is due to there being significant differences when considering the induced similarity order. Therefore, it can be concluded that ELICIT-t1-IOWA operator is more sensitive to different situations that are modeled by linguistic quantifiers in the ELICIT-I-IOWA operator. Hence, we can say that our proposal opens a way to deal with different views of solving GDM problems with ELICIT information in a more flexible way that could not be done previously.

Table 5. The comparative result with ELICIT expressions.

\begin{tabular}{ccc}
\hline Family of ELICIT-I-IOWA Operator & Ranking Result of F ELICIT-t1-IOWA & Ranking Result of F \\
\hline "moean \\
\hline "at least half" & $a_{4} \prec a_{1} \prec a_{2} \prec a_{3}$ & $a_{4} \prec a_{1} \prec a_{3} \prec a_{2}$ \\
\hline "as many as possible" & $a_{2} \prec a_{3} \prec a_{4} \prec a_{1}$ & $a_{4} \prec a_{2} \prec a_{1} \prec a_{3} \prec a_{2}$ \\
\hline "mean" & $a_{1} \prec a_{3} \prec a_{4} \prec a_{2}$ & $a_{4} \prec a_{3} \prec a_{1} \prec a_{2}$ \\
\hline
\end{tabular}

\section{Concluding Remarks}

In this article, when considering that the fuzzy representation of $\mathcal{F}$ has no inherent order, where $\mathcal{F}$ is the set of all possible ELICIT expressions, we developed the induced OWA (IOWA) operator for ELICIT information. Because the IOWA operator reorders the argument variable by the order inducing variable instead of the arguments themselves. For different types of weights, the operator based on the IOWA operator is also different for ELICIT information. For example, if the operator with the classical crisp weight is used, then it is called the ELICIT-IOWA operator. While considering fuzzy weights and type-1 OWA operators, first obtain the type-1 IOWA operator and then obtain the ELICITt1-IOWA operator according to Zadeh's extension principle. Because they are all based on the IOWA operator, they also have the properties of the classical IOWA operator, such as idempotency, commutativity, monotonicity, and boundedness. When considering that ELICIT information can be converted into trapezoidal fuzzy numbers, and that ELICIT information is intuitive, easy to understand, and explain, on this basis we can compute with the ELICIT information and also propose a proposal that the majority-driven GDM process be used for ELICIT information based on majority opinions. The aggregation of this process includes two aggregation stages. First, the ELICIT-t1-IOWA operator is used in order to aggregate with interval weights that are computed by the interval-valued type- 2 linguistic quantifier "most" to aggregate each preference relationship matrix represented by 
the ELICIT information. After that, based on each expert associated with the credibility and combined with the linguistic quantifier "most", where $\alpha=0.3, \beta=0.8$, to obtain the weight of the ELICIT-I-IOWA operator, the ELICIT-I-IOWA operator is used in order to obtain an overall result for each alternative. Finally, a briefly introduces the comparison among other linguistic quantifiers, such as "most", "at least half", "as many as possible", and "mean".

For future research, we will undertake research regarding the consensus reaching process (CRP) to group decision-making issues for handling ELICIT information, in which several experts believe that their opinions have not been considered.

Author Contributions: Conceptualization, W.H., R.M.R., B.D. and L.M.; methodology, W.H., B.D. and L.M.; validation, R.M.R., A.A.A. and L.M.; formal analysis, W.H., R.M.R. and B.D.; investigation, W.H., R.M.R. and L.M.; writing-original draft preparation, W.H., B.D. and L.M.; supervision, R.M.R., A.A.A. and L.M.; funding acquisition, R.M.R., A.A.A. and L.M. All authors have read and agreed to the published version of the manuscript.

Funding: This research was funded by the Deanship of Scientific Research (DSR), King Abdulaziz University, Jeddah, under Grant FP-125-42.

Conflicts of Interest: The authors declare no conflict of interest.

\section{Abbreviations}

The following abbreviations are used in this manuscript:

$\begin{array}{ll}\text { GDM } & \text { Group decision making } \\ \text { ELICIT } & \text { Extended comparative linguistic expressions with symbolic translation } \\ \text { HFLTSs } & \text { Hesitant fuzzy linguistic term sets } \\ \text { CLEs } & \text { Comparative Linguistic Expressions } \\ \text { OWA } & \text { Ordered weighted averaging } \\ \text { IOWA } & \text { Induced ordered weighted averaging } \\ \text { BUM } & \text { Basic unit-interval monotonic } \\ \text { C-OWA } & \text { Continuous ordered weighted averaging } \\ \text { CW } & \text { Computing with words } \\ \text { ELICIT-CW } & \text { Computing with words for ELICIT information } \\ \text { ELICIT-IOWA } & \text { Induced ordered weighted averaging aggregation over ELICIT information } \\ \text { ELICIT-I-IOWA } & \text { Important induced ordered weighted averaging aggregation over ELICIT } \\ & \text { information } \\ \text { FOU } & \text { Footprint of uncertainty } \\ \text { t1-IOWA } & \text { Type-1 induced ordered weighted averaging } \\ \text { ELICIT-t1-IOWA } & \text { Type-1 induced ordered weighted averaging aggregation over ELICIT } \\ & \text { information } \\ \text { WA } & \text { Weighted average } \\ \text { QFIOWA } & \text { Fuzzy induced quasi-arithmetic ordered weighted averaging } \\ \text { FIGOWA } & \text { Fuzzy induced generalized ordered weighted averaging } \\ \text { ELICIT-QIOWA } & \text { Induced quasi-arithmetic ordered weighted averaging aggregation over ELICIT } \\ \text { ELICIT-GIOWA } & \text { information } \\ & \text { Induced generalized ordered weighted averaging aggregation over ELICIT } \\ \text { EKM } & \text { information } \\ \text { CI } & \text { Enhanced Karnik-Mendel } \\ \text { NS } & \text { Centroid index } \\ \text { CRP } & \text { Numerical scale } \\ & \text { Consensus reaching process }\end{array}$




\section{References}

1. Lu, J.; Zhang, G.; Ruan, D.; Wu, F. Multi-Objective Group Decision Making; Imperial College Press: London, UK, 2006.

2. Rodríguez, R.; Martínez, L.; Herrera, F. A Group Decision Making Model dealing with Comparative Linguistic Expressions based on Hesitant Fuzzy Linguistic Term Sets. Inf. Sci. 2013, 241, 28-42. [CrossRef]

3. Pedrycz, W.; Ekel, P.; Parreiras, R. Fuzzy Multicriteria Decision-Making: Models, Methods and Applications; John Wiley \& Sons, Ltd.: Chichester, UK, 2010.

4. Chen, Z.S.; Zhang, X.; Rodríguez, R.M.; Wang, X.J.; Chin, K.S. Heterogeneous Interrelationships among Attributes in MultiAttribute Decision-Making: An Empirical Analysis. Int. J. Comput. Intell. Syst. 2019, 12, 984-997. [CrossRef]

5. Denoeux, T. Decision-making with belief functions: A review. Int. J. Approx. Reason. 2019, 109, 87-110. [CrossRef]

6. Pasman, H.J.; Rogers, W.J. How to treat expert judgment? With certainty it contains uncertainty! J. Loss Prev. Process. Ind. 2020, 66. [CrossRef]

7. Pelissari, R.; Oliveira, M.C.; Abackerli, A.J.; Ben-Amor, S.; Assumpcao, M.R.P. Techniques to model uncertain input data of multi-criteria decision-making problems: A literature review. Int. Trans. Oper. Res. 2021, 28, 523-559. [CrossRef]

8. Doukas, H. Linguistic multicriteria decision making for energy systems: building the 'RE2S' framework. Wires Energy Environ. 2013, 2, 571-585, [CrossRef]

9. Martínez, L.; Rodríguez, R.; Herrera, F. The 2-tuple Linguistic Model: Computing with Words in Decision Making; Springer: New York, NY, USA, 2015; p. 162.

10. Wang, L.; Wang, Y.; Martínez, L. Fuzzy TODIM method based on alpha-level sets. Expert Syst. Appl. 2020, 140, 112899. [CrossRef]

11. Yager, R. A new methodology for ordinal multiobjetive decision based on fuzzy sets. Decis. Sci. 1981, 12, 589-600. [CrossRef]

12. Zadeh, L.A. The concept of a linguistic variable and its application to approximate reasoning (I). Inf. Sci. 1975, 8, 199-249. [CrossRef]

13. Zadeh, L.A. The concept of a linguistic variable and its application to approximate reasoning (II). Inf. Sci. 1975, 8, 301-357. [CrossRef]

14. Zadeh, L.A. The concept of a linguistic variable and its application to approximate reasoning (III). Inf. Sci. 1975, 9, 43-80. [CrossRef]

15. Labella, Á.; Rodríguez, R.; Martínez, L. Computing with Comparative Linguistic Expressions and Symbolic Translation for Decision Making: ELICIT Information. IEEE Trans. Fuzzy Syst. 2019. [CrossRef]

16. Herrera, F.; Martínez, L. A 2-tuple Fuzzy Linguistic Representation Model for Computing with Words. IEEE Trans. Fuzzy Syst. 2000, 8, 746-752.

17. Rodríguez, R.; Martínez, L.; Herrera, F. Hesitant Fuzzy Linguistic Term Sets for Decision Making. IEEE Trans. Fuzzy Syst. 2012, 20, 1109-119. [CrossRef]

18. Zadeh, L. Fuzzy logic = computing with words. IEEE Trans. Fuzzy Syst. 1996, 4, 103-111. [CrossRef]

19. Mendel, J.; Zadeh, L.; Trillas, E.; Yager, R.; Lawry, J.; Hagras, H.; Guadarrama, S. What computing with words means to me: Discussion forum. IEEE Comput. Intell. Mag. 2010, 5, 20-26. [CrossRef]

20. Beliakov, G.; Pradera, A.; Calvo, T. Aggregation Functions: A Guide for Practitioners; Springer: Berlin/Heidelberg, Germany, 2007.

21. Dutta, B.; Labella, Á.; Rodríguez, R.; Martínez, L. Aggregating Interrelated Attributes in Multi-Attribute Decision-Making With ELICIT Information Based on Bonferroni Mean and Its Variants. Int. J. Comput. Intell. Syst. 2019, 12, 1179-1196. [CrossRef]

22. Filev, D.; Yager, R. On the issue of obtaining OWA operator weights. Fuzzy Sets Syst. 1998, 94, 157-169. [CrossRef]

23. Torra, V. The Weighted OWA Operator. Int. J. Intell. Syatems 1997, 12, 153-166. [CrossRef]

24. Yager, R. Families of OWA operators. Fuzzy Sets Syst. 1993, 59, 125-148. [CrossRef]

25. Grabisch, M.; Marichal, J.; Mesiar, R.; Pap, E. Aggregation Functions (Encyclopedia of Mathematics and Its Applications), 1st ed.; Cambridge University Press: New York, NY, USA, 2009.

26. Chen, S.J.; Chen, S.M. A new method for handling multicriteria fuzzy decision-making problems using FN-IOWA operators. Cybern. Syst. 2003, 34, 109-137. [CrossRef]

27. Yager, R.; Filev, D. Induced ordered weighted averaging operators. IEEE Trans. Syst. Man Cybern. Part -Cybern. 1999, 29, 141-150. [CrossRef]

28. Yager, R.R. Quantifier guided aggregation using OWA operators. Int. J. Intell. Syst. 1996, 11, 49-73. [CrossRef]

29. Pasi, G.; Yager, R. Modeling the concept of majority opinion in group decision making. Inf. Sci. 2006, 176, 390-414. [CrossRef]

30. Yager, R. On Ordered Weighted Averaging Aggregation Operators in Multicriteria Decisionmaking. IEEE Trans. Syst. Man Cybern. 1988, 18, 183-190. [CrossRef]

31. Yager, R. OWA aggregation over a continuous interval argument with applications to decision making. IEEE Trans. Syst. Man, Cybern. Part (Cybern.) 2004, 34, 1952-1963. [CrossRef]

32. Zhou, S.; Chiclana, F.; John, R.; Garibaldi, J. Type-1 OWA operators for aggregating uncertain information with uncertain weights induced by type-2 linguistic quantifiers. Fuzzy Sets Syst. 2008, 159, 3281-3296. [CrossRef]

33. Liu, H.; Rodríguez, R.M. A fuzzy envelope for hesitant fuzzy linguistic term set and its application to multicriteria decision making. Inf. Sci. 2014, 258, 220-238. [CrossRef]

34. Yager, R. Using trapezoids for representing granular objects: Applications to learning and OWA aggregation. Inf. Sci. 2008, 178, 363-380. [CrossRef]

35. Yager, R.R. Induced aggregation operators. Fuzzy Sets Syst. 2003, 137, 59-69. [CrossRef] 
36. Merigó, J.M.; Gil-Lafuente, A.M. Fuzzy induced generalized aggregation operators and its application in multi-person decision making. Expert Syst. Appl. 2011, 38, 9761-9772. [CrossRef]

37. Zhou, S.; Chiclana, F.; John, R.; Garibaldi, J. Alpha-level aggregation: A practical approach to type-1 OWA operation for aggregating uncertain information with applications to breast cancer treatments. IEEE Trans. Knowl. Data Eng. 2010, 23, 1455-1468. [CrossRef]

38. Mendel, J.M.; John, R.I.B. Type-2 fuzzy sets made simple. IEEE Trans. Fuzzy Syst. 2002, 10, 117-127. [CrossRef]

39. Mendel, J.M. Uncertain rule-based fuzzy systems. In Introduction and New Directions; Springer: Berlin/Heidelberg, Germany, 2017; p. 684.

40. Wu, D.; Mendel, J.M. Enhanced karnik-mendel algorithms. IEEE Trans. Fuzzy Syst. 2008, 17, $923-934$.

41. Rezvani, S.; Molani, M. Representation of trapezoidal fuzzy numbers with shape function. Ann. Fuzzy Math. Inform. 2014, 8, 89-112.

42. Wang, X.; Kerre, E.E. Reasonable properties for the ordering of fuzzy quantities (I). Fuzzy Sets Syst. 2001, 118, 375-385. [CrossRef]

43. Wang, X.; Kerre, E.E. Reasonable properties for the ordering of fuzzy quantities (II). Fuzzy Sets Syst. 2001, 118, 387-405. [CrossRef]

44. Yager, R. On choosing between fuzzy subsets. Kybernetes 1980, 9, 151-154 [CrossRef]

45. Abbasbandy, S.; Hajjari, T. A New Approach for Ranking of Trapezoidal Fuzzy Numbers. Comput. Math. Appl. 2009, 57, 413-419. [CrossRef]

46. Wang, X.; Ruan, D.; Kerre, E.E. Math. Fuzziness_Basic Issues; Springer: Berlin/Heidelberg, Germany, 2009 ; Volume 245.

47. Chen, S.H. Ranking fuzzy numbers with maximizing set and minimizing set. Fuzzy Sets Syst. 1985, 17, 113-129. [CrossRef]

48. Nakamura, K. Preference relations on a set of fuzzy utilities as a basis for decision making. Fuzzy Sets Syst. 1986, 20, 147-162. [CrossRef]

49. Yatsalo, B.I.; Martínez, L. Fuzzy rank acceptability analysis: A confidence measure of ranking fuzzy numbers. IEEE Trans. Fuzzy Syst. 2018, 26, 3579-3593. [CrossRef]

50. Dong, Y.; Xu, Y.; Yu, S. Computing the numerical scale of the linguistic term set for the 2-tuple fuzzy linguistic representation model. IEEE Trans. Fuzzy Syst. 2009, 17, 1366-1378. [CrossRef]

51. Mendel, J. The perceptual computer: An architecture for computing with words. IEEE Int. Conf. Fuzzy Syst. 2001, 1, 35-38.

52. Roubens, M. Fuzzy sets and decision analysis. Fuzzy Sets Syst. 1997, 90, 199-206. [CrossRef]

53. Yager, R.R. The power average operator. IEEE Trans. Syst. Man, -Cybern.-Part Syst. Humans 2001, 31, 724-731. [CrossRef] 